\title{
A priori error for unilateral contact problems with Lagrange multipliers and IsoGeometric Analysis
}

\author{
Pablo Antolin*, Annalisa Buffa*, ${ }^{*}$, Mathieu Fabre*,†
}

\begin{abstract}
In this paper, we consider unilateral contact problem without friction between a rigid body and deformable one in the framework of isogeometric analysis. We present the theoretical analysis of the mixed problem. For the displacement, we use the pushforward of a NURBS space of degree $p$ and for the Lagrange multiplier, the pushforward of a B-Spline space of degree $p-2$. These choices of space ensure to prove an inf - sup condition and so on, the stability of the method. We distinguish between contact and non-contact sets to avoid of classical geometrical hypothesis of the contact set. An optimal a priori error estimate is demonstrated without assumption on the unknown contact set. Several numerical examples in two- and three-dimensions and in small and large deformation demonstrate the accuracy of the proposed method.
\end{abstract}

\section{Introduction}

In the past few years, the study of contact problems in small and large deformation is increased. The numerical solution of contact problems presents several difficulties as the computational cost, the high nonlinearity and the ill-conditioning. Contrary to many other problems in nonlinear mechanics, these problems can not be solved always at a satisfactory level of robustness and accuracy $[19,28]$ with the introduce numerical methods.

One of the reasons that make robustness and accuracy hard to achieve is that the computation of gap, i.e. the distance between the deformed body and the obstacle is indeed an ill-posed problem and its numerical approximation often introduce extra discontinuity that breaks the converge of the iterative schemes; see $[1,19,28,18,23]$ where a master-slave method is introduced to weaken this effect.

To this respect, the use of NURBS or spline approximations within the framework of isogeometric analysis [17], holds great promises thanks to the increased regularity in the geometric description which makes the gap computation intrinsically easier. The IGA-based methods use a generalization of Bézier's curves, the B-Splines and non-uniform rational B-Splines (NURBS). These functions, used to represent the geometry of the domains with CAD, are used as basis functions to approximate a partial differential equations, it is called the isoparametric paradigm. The smooth IGA basis functions possess a number of signifiant advantages for the analysis, including exact geometry and superior approximation. Isogeometric methods for frictionless contact problems have been introduced in $[29,25,26,10,9,8]$, see also with primal and dual elements

\footnotetext{
*EPFL SB MATHICSE MNS (Bât. MA), station 8, CH 1015 Lausanne (Switzerland).

†Istituto di Matematica Applicata e Tecnologie Informatiche 'E. Magenes' del CNR via Ferrata 1, 27100, Pavia (Italy).

email: pablo.antolin@epfl.ch, annalisa.buffa@epfl.ch, mathieu.fabre@epfl.ch.
} 
$[27,16,15,22,24]$. Both point-to-segment and segment-to-segment (i.e, mortar type) algorithms have been designed and tested with an engineering perspective, showing that, indeed, the use of smooth geometric representation helps the design of reliable methods for contact problems.

In this paper, we take a slightly different point of view. Inspired by the recent design and analysis of isogeometric mortar methods in [6], we consider a formulation of frictionless contact based on the choice of the Lagrange multiplier space proposed there. Indeed, we associate to NURBS displacement of degree $p$, a space of Lagrange multiplier of degree $p-2$. The use of lower order multipliers has several advantages because it makes the evaluation of averaged gap values at active and inactive control points simpler, accurate and substantially more local. This choice of multipliers is then coupled with an active-set strategy, as the one proposed and used in $[16,15]$.

Moreover, it is supposed classically, for the exact solution $u$, it existed a finite number of points where the transition between contact and non-contact occurs. In the article [11], a contact set and non-contact set for the displacement are defined for Signorini problem. This technic allow to avoid an additional assumption of the unknown contact set to prove an a priori error estimate.

Finally, we perform a comprehensive set of tests both in small and large scale deformation, which well show the performance of our method. These tests have been performed with an in-house code developed upon the public library igatools [21].

The outline of the paper is structured as follows in Section 1, we introduce unilateral contact problem, some notations. In Section 2, we describes the discrete spaces and their properties. In Section 3, we present the theoretical analysis of the mixed problem. An optimal a priori error estimate without assumption on the unknown contact set is presented. In the last section, some two- and three-dimensional problem in small deformation are presented in order to illustrate the convergence of the method with active-set strategy. A two-dimensional problem in large deformation with Neo-Hookean material law is provided to show the robustness of this method.

Remark. The letter $C$ stands for a generic constant, independent of the discretization parameters and the solution $u$ of the variational problem. For two scalar quantities a and $b$, the notation $a \lesssim b$ means there exists a constant $C$, independent of the mesh size parameters, such that $a \leq C b$. Moreover, $a \sim b$ means that $a \lesssim b$ and $b \lesssim a$.

\section{Preliminaries and notations}

\subsection{Unilateral contact problem}

Let $\Omega \subset \mathbb{R}^{d}$ ( $d=2$ or 3 ) be a bounded regular domain which represents the reference configuration of a body submitted to a Dirichlet condition on $\Gamma_{D}$ (with meas $\left(\Gamma_{D}\right)>0$ ), a Neumann condition on $\Gamma_{N}$ and a unilateral contact condition on a potential zone of contact $\Gamma_{C}$ with a rigid body. Without loss of generality, it is assumed that the body is subjected to a volume force $f$, to a surface traction $\ell$ on $\Gamma_{N}$ and clamped at $\Gamma_{D}$. Finally, we denote by $n_{\Omega}$ the unit outward normal vector on $\partial \Omega$.

In what follows, we call $u$ the displacement of $\Omega, \varepsilon(u)=\frac{1}{2}\left(\nabla u+\nabla u^{T}\right)$ its linearized strain tensor and we denote by $\sigma=\left(\sigma_{i j}\right)_{1 \leq i, j \leq d}$ the stress tensor. We assume a linear constitutive law between $\sigma$ and $\varepsilon$, i.e. $\sigma(u)=A \varepsilon(u)$, where $A=\left(a_{i j k l}\right)_{1 \leq i, j, k, l \leq d}$ is a fourth order symmetric tensor verifying the usual bounds:

$$
\text { - } a_{i j k l} \in L^{\infty}(\Omega) \text {, i.e. there exists a constant } m \text { such that } \max _{1 \leq i, j, k, l \leq d}\left|a_{i j k l}\right| \leq m \text {; }
$$


- there exists a constant $M>0$ such that a.e. on $\Omega$,

$$
a_{i j k l} \varepsilon_{i j} \varepsilon_{k l} \geq M \varepsilon_{i j} \varepsilon_{i j} \quad \forall \varepsilon \in \mathbb{R}^{d \times d} \text { with } \varepsilon_{i j}=\varepsilon_{j i} .
$$

Let $n$ be the outward unit normal vector at the rigid body. From now on, we assume that $n$ is an infinitely regular field. For any displacement field $u$ and for any density of surface forces $\sigma(u) n$ defined on $\partial \Omega$, we adopt the following notation:

$$
u=u_{n} n+u_{t} \quad \text { and } \quad \sigma(u) n=\sigma_{n}(u) n+\sigma_{t}(u),
$$

where $u_{t}$ (resp. $\left.\sigma_{t}(u)\right)$ are the tangential components with respect to $n$.

The unilateral contact problem between a rigid body and the elastic body $\Omega$ consists in finding the displacement $u$ satisfying:

$$
\begin{aligned}
\operatorname{div} \sigma(u)+f & =0 & & \text { in } \Omega, \\
\sigma(u) & =A \varepsilon(u) & & \text { in } \Omega, \\
u & =0 & & \text { on } \Gamma_{D}, \\
\sigma(u) n_{\Omega} & =\ell & & \text { on } \Gamma_{N} .
\end{aligned}
$$

and the conditions describing unilateral contact without friction at $\Gamma_{C}$ are:

$$
\begin{aligned}
u_{n} \geq 0 & (i), \\
\sigma_{n}(u) \leq 0 & (i i), \\
\sigma_{n}(u) u_{n}=0 & (i i i), \\
\sigma_{t}(u)=0 & (i v) .
\end{aligned}
$$

In order to describe the variational formulation of (1)-(2), we consider the Hilbert spaces:

$$
V:=H_{0, \Gamma_{D}}^{1}(\Omega)^{d}=\left\{v \in H^{1}(\Omega)^{d}, \quad v=0 \text { on } \Gamma_{D}\right\}, \quad W=\left\{\left.v_{n}\right|_{\Gamma_{C}}, \quad v \in V\right\},
$$

and their dual spaces $V^{\prime}, W^{\prime}$ endowed with their usual norms. We denote by:

$$
\|v\|_{V}=\left(\|v\|_{L^{2}(\Omega)^{d}}^{2}+|v|_{H^{1}(\Omega)^{d}}^{2}\right)^{1 / 2}, \forall v \in V .
$$

If $\bar{\Gamma}_{D} \cap \bar{\Gamma}_{C}=\emptyset$ and $n$ is regular enough, it is well known that $W=H^{1 / 2}\left(\Gamma_{C}\right)$ and we denote $W^{\prime}$ by $H^{-1 / 2}\left(\Gamma_{C}\right)$. On the other hand, if $\bar{\Gamma}_{D} \cap \bar{\Gamma}_{C} \neq \emptyset$, it will hold that $H_{00}^{1 / 2}\left(\Gamma_{C}\right) \subset W \subset H^{1 / 2}\left(\Gamma_{C}\right)$. In all cases, we will denote by $\|\cdot\|_{W}$ the norm on $W$ and by $\langle\cdot, \cdot\rangle_{W^{\prime}, W}$ the duality pairing between $W^{\prime}$ and $W$.

For all $u$ and $v$ in $V$, we set:

$$
a(u, v)=\int_{\Omega} \sigma(u): \varepsilon(v) \mathrm{d} \Omega \quad \text { and } \quad L(v)=\int_{\Omega} f \cdot v \mathrm{~d} \Omega+\int_{\Gamma_{N}} \ell \cdot v \mathrm{~d} \Gamma .
$$

Let $K_{C}$ be the closed convex cone of admissible displacement fields satisfying the non-interpenetration conditions, $K_{C}:=\left\{v \in V, \quad v_{n} \geq 0\right.$ on $\left.\Gamma_{C}\right\}$. A weak formulation of Problem (1)-(2) (see [20]), as a variational inequality, is to find $u \in K_{C}$ such as:

$$
a(u, v-u) \geq L(v-u), \quad \forall v \in K_{C} .
$$

We cannot directly use a Newton-Raphson's method to solve the formulation (3). A classical solution is to introduce a new variable, the Lagrange multipliers denoted by $\lambda$, which represents 
the surface normal force. For all $\lambda$ in $W^{\prime}$, we denote $b(\lambda, v)=-\left\langle\lambda, v_{n}\right\rangle_{W^{\prime}, W}$ and $M$ is the classical convex cone of multipliers on $\Gamma_{C}$ :

$$
M:=\left\{\mu \in W^{\prime}, \quad\langle\mu, \psi\rangle_{W^{\prime}, W} \leq 0 \quad \forall \psi \in H^{1 / 2}\left(\Gamma_{C}\right), \quad \psi \geq 0 \text { a.e. on } \Gamma_{C}\right\} .
$$

The complementary conditions with Lagrange multipliers writes as follows:

$$
\begin{aligned}
u_{n} \geq 0 & (i) \\
\lambda \leq 0 & (i i) \\
\lambda u_{n}=0 & (i i i)
\end{aligned}
$$

The mixed formulation [4] of the Signorini problem (1) and (4) consists in finding $(u, \lambda) \in V \times M$ such that:

$$
\left\{\begin{aligned}
a(u, v)-b(\lambda, v)=L(v), & \forall v \in V, \\
b(\mu-\lambda, u) \geq 0, & \forall \mu \in M .
\end{aligned}\right.
$$

Stampacchia's Theorem ensures that problem (5) admits a unique solution.

The existence and uniqueness of the solution $(u, \lambda)$ of the mixed formulation has been established in [12] and it holds $\lambda=\sigma_{n}(u)$.

To simplify the notation, we denote by $\|\cdot\|_{3 / 2+s, \Omega}$ the norm on $H^{3 / 2+s}(\Omega)^{d}$ and by $\|\cdot\|_{s, \Gamma_{C}}$ the norm on $H^{s}\left(\Gamma_{C}\right)$.

So, the following classical inequality (see [2]) holds:

Theorem 1.1. Given $s>0$, if the displacement $u$ verifies $u \in H^{3 / 2+s}(\Omega)^{d}$, then $\lambda \in H^{s}\left(\Gamma_{C}\right)$ and it holds:

$$
\|\lambda\|_{s, \Gamma_{C}} \leq\|u\|_{3 / 2+s, \Omega} .
$$

The aim of this paper is to discretize the problem (5) within the isogeometric paradigm, i.e. with splines and NURBS. Moreover, in order to properly choose the space of Lagrange multipliers, we will be inspired by [6]. In what follows, we introduce NURBS spaces and assumptions together with relevant choices of space pairings. In particular, following [6], we concentrate on the definitions of B-Splines displacements of degree $p$ and multiplier spaces of degree $p-2$.

\section{$1.2 \quad$ NURBS discretisation}

In this section, we describe briefly an overview on isogeometric analysis providing the notation and concept needed in the next sections. Firstly, we define B-Splines and NURBS in one-dimension. Secondly, we extend these definitions to the multi-dimensional case. Finally, we define the primal and the dual spaces for the contact boundary.

Let us denote by $p$ the degree of univariate B-Splines and by $\Xi$ an open univariate knot vector, where the first and last entries are repeated $(p+1)$-times, i.e.

$$
\Xi:=\left\{0=\xi_{1}=\cdots=\xi_{p+1}<\xi_{p+2} \leq \cdots \leq \xi_{\eta}<\xi_{\eta+1}=\cdots=\xi_{\eta+p+1}\right\}
$$

Let us define $Z=\left\{\zeta_{1}, \ldots, \zeta_{E}\right\}$ as vector of breakpoints, i.e. knots taken without repetition, and $m_{j}$, the multiplicity of the breakpoint $\xi_{j}, j=1, \ldots, E$. Let $\Xi$ be the open knot vector associated to $Z$ where each breakpoint is repeated $m_{j}$-times, i.e. In what follows, we suppose that $m_{1}=m_{E}=p+1$, while $m_{j} \leq p-1, \forall j=2, \ldots, E-1$. We define by $\hat{B}_{i}^{p}(\zeta), i=1, \ldots, \eta$ the $i$-th univariable B-Spline based on the univariate knot vector $\Xi$ and the degree $p$. We denote 
by $S^{p}(\Xi)=\operatorname{Span}\left\{\hat{B}_{i}^{p}(\zeta), i=1, \ldots, \eta\right\}$. Moreover, for further use we denote by $\tilde{\Xi}$ the sub-vector of $\Xi$ obtained by removing the first and the last knots.

Multivariate B-Splines in dimension $d$ are obtained by tensor product of univariate B-Splines. For any direction $\delta \in\{1, \ldots, d\}$, we define by $\eta_{\delta}$ the number of B-Splines, $\Xi_{\delta}$ the open knot vector and $Z_{\delta}$ the breakpoint vector. Then, we define the multivariate knot vector by $\boldsymbol{\Xi}=\left(\Xi_{1} \times \ldots \times \Xi_{d}\right)$ and the multivariate breakpoint vector by $\boldsymbol{Z}=\left(Z_{1} \times \ldots \times Z_{d}\right)$. We introduce a set of multiindices $\boldsymbol{I}=\left\{\boldsymbol{i}=\left(i_{1}, \ldots, i_{d}\right) \mid 1 \leq i_{\delta} \leq \eta_{\delta}\right\}$. We build the multivariate B-Spline functions for each multi-index $\boldsymbol{i}$ by tensorization from the univariate B-Splines, let $\boldsymbol{\zeta} \in \boldsymbol{Z}$ be a parametric coordinate of the generic point:

$$
\hat{B}_{i}^{p}(\boldsymbol{\zeta})=\hat{B}_{i_{1}}^{p}\left(\zeta_{1}\right) \ldots \hat{B}_{i_{d}}^{p}\left(\zeta_{d}\right)
$$

Let us define the multivariate spline space in the reference domain by (for more details, see [6]):

$$
S^{p}(\boldsymbol{\Xi})=\operatorname{Span}\left\{\hat{B}_{\boldsymbol{i}}^{p}(\boldsymbol{\zeta}), \boldsymbol{i} \in \boldsymbol{I}\right\} .
$$

We define $N^{p}(\boldsymbol{\Xi})$ as the NURBS space, spanned by the function $\hat{N}_{\boldsymbol{i}}^{p}(\boldsymbol{\zeta})$ with

$$
\hat{N}_{i}^{p}(\boldsymbol{\zeta})=\frac{\omega_{\boldsymbol{i}} \hat{B}_{\boldsymbol{i}}^{p}(\boldsymbol{\zeta})}{\hat{W}(\boldsymbol{\zeta})}
$$

where $\left\{\omega_{\boldsymbol{i}}\right\}_{\boldsymbol{i} \in \boldsymbol{I}}$ is a set of positive weights and $\hat{W}(\boldsymbol{\zeta})=\sum_{\boldsymbol{i} \in \boldsymbol{I}} \omega_{\boldsymbol{i}} \hat{B}_{\boldsymbol{i}}^{p}(\boldsymbol{\zeta})$ is the weight function and we set

$$
N^{p}(\boldsymbol{\Xi})=\operatorname{Span}\left\{\hat{N}_{\boldsymbol{i}}^{p}(\boldsymbol{\zeta}), \boldsymbol{i} \in \boldsymbol{I}\right\} .
$$

In what follows, we will assume that $\Omega$ is obtained as image of $\hat{\Omega}=] 0,1{ }^{d}$ through a NURBS mapping $\varphi_{0}$, i.e. $\Omega=\varphi_{0}(\hat{\Omega})$. Moreover, in order to simplify our presentation, we assume that $\Gamma_{C}$ is the image of a full face $\hat{f}$ of $\hat{\Omega}$, i.e. $\Gamma_{C}=\varphi_{0}(\hat{f})$. We denote by $\varphi_{0, \Gamma_{C}}$ the restriction of $\varphi_{0}$ to $\hat{f}$.

A NURBS surface, in $d=2$, or solid, in $d=3$, is parameterised by

$$
\mathcal{C}(\boldsymbol{\zeta})=\sum_{i \in \boldsymbol{I}} C_{\boldsymbol{i}} \hat{N}_{i}^{p}(\boldsymbol{\zeta})
$$

where $C_{\boldsymbol{i} \in \boldsymbol{I}} \in \mathbb{R}^{d}$, is a set of control point coordinates. The control points are somewhat analogous to nodal points in finite element analysis. The NURBS geometry is defined as the image of the reference domain $\hat{\Omega}$ by $\varphi$, called geometric mapping, $\Omega_{t}=\varphi(\hat{\Omega})$.

We remark that the physical domain $\Omega$ is split into elements by the image of $\boldsymbol{Z}$ through the map $\varphi_{0}$. We denote such a physical mesh $\mathcal{Q}_{h}$ and physical elements in this mesh will be called $Q . \Gamma_{C}$ inherits a mesh that we denote by $\left.\mathcal{Q}_{h}\right|_{\Gamma_{C}}$. Elements on this mesh will be denoted as $Q_{C}$.

Finally, we introduce some notations and assumptions on the mesh.

Assumption 1. The mapping $\varphi_{0}$ is considered to be a bi-Lipschitz homeomorphism. Furthermore, for any parametric element $\hat{Q},\left.\varphi_{0}\right|_{\hat{Q}}$ is in $\mathcal{C}^{\infty}(\overline{\hat{Q}})$ and for any physical element $Q,\left.\varphi_{0}^{-1}\right|_{\bar{Q}}$ is in $\mathcal{C}^{\infty}(\bar{Q})$.

Let $h_{Q}$ be the size of an physical element $Q$, it holds $h_{Q}=\operatorname{diam}(Q)$. In the same way, we define the mesh size for any parametric element. In addition, the Assumption 1 ensures that both size of mesh are equivalent. We denote the maximal mesh size by $h=\max _{Q \in \mathcal{Q}_{h}} h_{Q}$. 
Assumption 2. The mesh $\mathcal{Q}_{h}$ is quasi-uniform, i.e there exists a constant $\theta$ such that $\frac{h_{Q}}{h_{Q^{\prime}}} \leq \theta$ with $Q$ and $Q^{\prime} \in \mathcal{Q}_{h}$.

\section{Discrete spaces and their properties}

We concentrate now on the definition of spaces on the domain $\Omega$.

For displacements, we denote by $V^{h} \subset V$ the space of mapped NURBS of degree $p$ with appropriate homogeneous Dirichlet boundary condition:

$$
V^{h}:=\left\{v^{h}=\hat{v}^{h} \circ \varphi_{0}^{-1}, \quad \hat{v}^{h} \in N^{p}(\boldsymbol{\Xi})^{d}\right\} \cap V .
$$

We denote the space of traces normal to the rigid body as:

$$
W^{h}:=\left\{\psi^{h}, \quad \exists v^{h} \in V^{h}: \quad v^{h} \cdot n=\psi^{h} \text { on } \Gamma_{C}\right\} .
$$

For multipliers, following the ideas of [6], we define the space of B-Splines of degree $p-2$ on the potential contact zone $\Gamma_{C}=\varphi_{0, \Gamma_{C}}(\hat{f})$. We denote by $\boldsymbol{\Xi}_{\hat{f}}$ the knot vector defined on $\hat{f}$ and by $\tilde{\boldsymbol{\Xi}}_{\hat{f}}$ the knot vector obtained by removing the first and last value in each knot vector. We define:

$$
\Lambda^{h}:=\left\{\lambda^{h}=\hat{\lambda}^{h} \circ \varphi_{0, \Gamma_{C}}^{-1}, \quad \hat{\lambda}^{h} \in S^{p-2}\left(\tilde{\boldsymbol{\Xi}}_{\hat{f}}\right)\right\} .
$$

The scalar space $\Lambda^{h}$ is spanned by mapped B-Splines of the type $\hat{B}_{\boldsymbol{i}}^{p-2}(\boldsymbol{\zeta}) \circ \varphi_{0, \Gamma_{C}}^{-1}$ for $\boldsymbol{i}$ belonging to a suitable set of indices. In order to reduce our notation, we call $K$ the unrolling of the multi-index $\boldsymbol{i}, K=0 \ldots \mathcal{K}$ and remove super-indices: for $K$ corresponding a given $\boldsymbol{i}$, we set $\hat{B}_{K}(\boldsymbol{\zeta})=\hat{B}_{\boldsymbol{i}}^{p-2}(\boldsymbol{\zeta}), B_{K}=\hat{B}_{K} \circ \varphi_{0, \Gamma_{C}}^{-1}$ and:

$$
\Lambda^{h}:=\operatorname{Span}\left\{B_{K}(x), \quad K=0 \ldots \mathcal{K}\right\} .
$$

For further use, for $v \in L^{2}\left(\Gamma_{C}\right)$ and for each $K=0 \ldots \mathcal{K}$, we denote by $\left(\Pi_{\lambda^{*}}^{h}\right)_{K}$ the following weighted average of $v$ :

$$
\left(\Pi_{\lambda}^{h} v\right)_{K}=\frac{\int_{\Gamma_{C}} v B_{K} \mathrm{~d} \Gamma}{\int_{\Gamma_{C}} B_{K} \mathrm{~d} \Gamma},
$$

and by $\Pi_{\lambda}^{h}$ the global operator such as:

$$
\Pi_{\lambda}^{h} v=\sum_{K=0}^{\mathcal{K}}\left(\Pi_{\lambda}^{h} v\right)_{K} B_{K} .
$$

We denote by $L^{h}$ the subset of $W^{h}$ on which the non-negativity holds only at the control points:

$$
L^{h}=\left\{\varphi^{h} \in W^{h}, \quad\left(\Pi_{\lambda}^{h} \varphi^{h}\right)_{K} \geq 0 \quad \forall K\right\} .
$$

We note that $L^{h}$ is a convex subset of $W^{h}$.

Next, we define the discrete space of the Lagrange multipliers as the negative cones of $L^{h}$ by

$$
M^{h}:=L^{h, *}=\left\{\mu^{h} \in \Lambda^{h}, \quad \int_{\Gamma_{C}} \mu^{h} \varphi^{h} \mathrm{~d} \Gamma \leq 0 \quad \forall \varphi^{h} \in L^{h}\right\} .
$$


For any $\left.Q_{C} \in \mathcal{Q}_{h}\right|_{\Gamma_{C}}, \tilde{Q}_{C}$ denotes the support extension of $Q_{C}$ (see $\left.[2,3]\right)$ defined as the image of supports of B-Splines that are not zero on $\hat{Q}_{C}=\varphi_{0, \Gamma_{C}}^{-1}\left(Q_{C}\right)$.

We notice that the operator verifies the following estimate error:

Lemma 2.1. Let $\psi \in H^{s}\left(\Gamma_{C}\right)$ with $0 \leq s \leq 1$, the estimate for the local interpolation error reads:

$$
\left\|\psi-\Pi_{\lambda}^{h}(\psi)\right\|_{0, Q_{C}} \lesssim h^{s}\|\psi\|_{s, \tilde{Q}_{C}},\left.\quad \forall Q_{C} \in \mathcal{Q}_{h}\right|_{\Gamma_{C}}
$$

Proof: First, Let $c$ be a constant. It holds:

$$
\Pi_{\lambda}^{h} c=\sum_{K=0}^{\mathcal{K}}\left(\Pi_{\lambda}^{h} c\right)_{K} B_{K}=\sum_{K=0}^{\mathcal{K}} \frac{\int_{\Gamma_{C}} c B_{K} \mathrm{~d} \Gamma}{\int_{\Gamma_{C}} B_{K} \mathrm{~d} \Gamma} B_{K}=\sum_{K=0}^{\mathcal{K}} c \frac{\int_{\Gamma_{C}} B_{K} \mathrm{~d} \Gamma}{\int_{\Gamma_{C}} B_{K} \mathrm{~d} \Gamma} B_{K}=c \sum_{K=0}^{\mathcal{K}} B_{K} .
$$

Using that B-Splines are a partition of the unity, we obtain $\Pi_{\lambda}^{h} c=c$.

Let $\psi \in H^{s}\left(\Gamma_{C}\right)$, it holds:

$$
\begin{aligned}
\left\|\psi-\Pi_{\lambda}^{h}(\psi)\right\|_{0, Q_{C}} & =\left\|\psi-c-\Pi_{\lambda}^{h}(\psi-c)\right\|_{0, Q_{C}} \\
& \leq\|\psi-c\|_{0, Q_{C}}+\left\|\Pi_{\lambda}^{h}(\psi-c)\right\|_{0, Q_{C}}
\end{aligned}
$$

We need now to bound the operator $\Pi_{\lambda}^{h}$. We obtain:

$$
\begin{aligned}
\left\|\Pi_{\lambda}^{h}(\psi-c)\right\|_{0, Q_{C}} & =\left\|\sum_{K=0}^{\mathcal{K}} \frac{\int_{\Gamma_{C}}(\psi-c) B_{K} \mathrm{~d} \Gamma}{\int_{\Gamma_{C}} B_{K} \mathrm{~d} \Gamma} B_{K}\right\|_{0, Q_{C}} \\
& \leq \sum_{K: \operatorname{supp} B_{K} \cap Q_{C} \neq \emptyset}\left|\frac{\int_{\Gamma_{C}}(\psi-c) B_{K} \mathrm{~d} \Gamma}{\int_{\Gamma_{C}} B_{K} \mathrm{~d} \Gamma}\right|\left\|B_{K}\right\|_{0, Q_{C}} \\
& \leq \sum_{K: \operatorname{supp} B_{K} \cap Q_{C} \neq \emptyset}^{\mathcal{K}}\|(\psi-c)\|_{0, \tilde{Q}_{C}} \frac{\left\|B_{K}\right\|_{0, \tilde{Q}_{C}}}{\int_{\Gamma_{C}} B_{K} \mathrm{~d} \Gamma}\left\|B_{K}\right\|_{0, Q_{C}} .
\end{aligned}
$$

Using $\left\|B_{K}\right\|_{0, \tilde{Q}_{C}} \sim\left|\tilde{Q}_{C}\right|^{1 / 2},\left\|B_{K}\right\|_{0, Q_{C}} \sim\left|Q_{C}\right|^{1 / 2}, \int_{\Gamma_{C}} B_{K} \mathrm{~d} \Gamma \sim\left|\tilde{Q}_{C}\right|$ and Assumption 1, it holds:

$$
\left\|\Pi_{\lambda}^{h}(\psi-c)\right\|_{0, Q_{C}} \lesssim\|(\psi-c)\|_{0, \tilde{Q}_{C}}
$$

Using the previous inequalities (11) and (12), for $0 \leq s \leq 1$, we obtain:

$$
\left\|\psi-\Pi_{\lambda}^{h}(\psi)\right\|_{0, Q_{C}} \lesssim\|\psi-c\|_{0, \tilde{Q}_{C}} \lesssim h_{\tilde{Q}_{C}}^{s}|\psi|_{s, \tilde{Q}_{C}}
$$

Proposition 2.2. For $h$ sufficiently small, there exists a $\beta>0$ such that:

$$
\inf _{\mu^{h} \in M^{h}} \sup _{\psi^{h} \in W^{h}} \frac{-\int_{\Gamma_{C}} \psi^{h} \mu^{h} \mathrm{~d} \Gamma}{\left\|\psi^{h}\right\|_{0, \Gamma_{C}}\left\|\mu^{h}\right\|_{0, \Gamma_{C}}} \geq \beta .
$$


Proof: In the article [6], the authors prove that, if $h$ is sufficiently small, there exists a constant $\beta$ independent of $h$ such that:

$$
\forall \phi^{h} \in\left(\Lambda^{h}\right)^{d},\left.\quad \exists u^{h} \in V^{h}\right|_{\Gamma_{C}}, \quad \text { s.t. } \quad \frac{-\int_{\Gamma_{C}} \phi^{h} \cdot u^{h} \mathrm{~d} \Gamma}{\left\|u^{h}\right\|_{0, \Gamma_{C}}} \geq \beta\left\|\phi^{h}\right\|_{0, \Gamma_{C}} .
$$

Given now a $\lambda^{h} \in \Lambda^{h}$ and $\psi^{h} \in W^{h}$, we should like to choose $\phi^{h}=\lambda^{h} n$ and $\psi^{h}=u^{h} \cdot n$ in (14), but, unfortunately, it is clear that $\phi^{h} \notin\left(\Lambda^{h}\right)^{d}$. Indeed, (13) can obtained from (14) via a superconvergence argument that we discuss in the next lines.

Let $\Pi_{\left(\Lambda^{h}\right)^{d}}: L^{2}\left(\Gamma_{C}\right)^{d} \rightarrow\left(\Lambda^{h}\right)^{d}$ be a quasi-interpolant defined and studied in e.g. see [3].

If $n \in W^{p-1, \infty}\left(\Gamma_{C}\right)$, by the same super-convergence argument used in [6], we obtain that:

$$
\left\|\phi^{h}-\Pi_{\left(\Lambda^{h}\right)^{d}}\left(\phi^{h}\right)\right\|_{0, \Gamma_{C}} \leq \alpha h\left\|\phi^{h}\right\|_{0, \Gamma_{C}} .
$$

Note that:

$$
\begin{aligned}
b\left(\lambda^{h}, u^{h}\right) & =-\int_{\Gamma_{C}} \lambda^{h}\left(u^{h} \cdot n\right) \mathrm{d} \Gamma=-\int_{\Gamma_{C}} \phi^{h} \cdot u^{h} \mathrm{~d} \Gamma \\
& =-\int_{\Gamma_{C}} \Pi_{\left(\Lambda^{h}\right)^{d}}\left(\phi^{h}\right) \cdot u^{h} \mathrm{~d} \Gamma-\int_{\Gamma_{C}}\left(\phi^{h}-\Pi_{\left(\Lambda^{h}\right)^{d}}\left(\phi^{h}\right)\right) \cdot u^{h} \mathrm{~d} \Gamma .
\end{aligned}
$$

By inf - sup condition (14), we get:

$$
\sup _{u^{h} \in V^{h}} \frac{-\int_{\Gamma_{C}} \Pi_{\left(\Lambda^{h}\right)^{d}}\left(\phi^{h}\right) \cdot u^{h} \mathrm{~d} \Gamma}{\left\|u^{h}\right\|_{0, \Gamma_{C}}} \geq \beta\left\|\Pi_{\left(\Lambda^{h}\right)^{d}}\left(\phi^{h}\right)\right\|_{0, \Gamma_{C}}
$$

By (15), it holds:

$$
\int_{\Gamma_{C}}\left(\phi^{h}-\Pi_{\left(\Lambda^{h}\right)^{d}}\left(\phi^{h}\right)\right) \cdot u^{h} \mathrm{~d} \Gamma \leq \alpha h\left\|\phi^{h}\right\|_{0, \Gamma_{C}}\left\|u^{h}\right\|_{0, \Gamma_{C}}
$$

Thus:

$$
\frac{b\left(\lambda^{h}, u^{h}\right)}{\left\|u^{h}\right\|_{0, \Gamma_{C}}} \geq \beta\left\|\Pi_{\left(\Lambda^{h}\right)^{d}}\left(\phi^{h}\right)\right\|_{0, \Gamma_{C}}-\alpha h\left\|\phi^{h}\right\|_{0, \Gamma_{C}} .
$$

Noting that $\left\|\Pi_{\left(\Lambda^{h}\right)^{d}}\left(\phi^{h}\right)\right\|_{0, \Gamma_{C}} \geq\left\|\phi^{h}\right\|_{0, \Gamma_{C}}-\alpha h\left\|\phi^{h}\right\|_{0, \Gamma_{C}},\left\|\phi^{h}\right\|_{0, \Gamma_{C}} \sim\left\|\lambda^{h}\right\|_{0, \Gamma_{C}}$ and $\left\|u^{h}\right\|_{0, \Gamma_{C}} \sim$ $\left\|\psi^{h}\right\|_{0, \Gamma_{C}}$. Finally, we obtain:

$$
\sup _{u^{h} \in V^{h}} \frac{-\int_{\Gamma_{C}} \psi^{h} \lambda^{h} \mathrm{~d} \Gamma}{\left\|\psi^{h}\right\|_{0, \Gamma_{C}}} \geq \beta\left\|\lambda^{h}\right\|_{0, \Gamma_{C}}-\alpha h\left\|\lambda^{h}\right\|_{0, \Gamma_{C}} .
$$

For $h$ is sufficiently small, this implies that there exists a constant $\beta^{\prime}$ independent of $h$ such that:

$$
\sup _{u^{h} \in V^{h}} \frac{-\int_{\Gamma_{C}} \psi^{h} \lambda^{h} \mathrm{~d} \Gamma}{\left\|\psi^{h}\right\|_{0, \Gamma_{C}}} \geq \beta^{\prime}\left\|\lambda^{h}\right\|_{0, \Gamma_{C}}
$$


Lemma 2.3. For $h$ is sufficiently small, $M^{h}$ can be characterised as follow:

$$
M^{h} \equiv\left\{\mu^{h} \in \Lambda^{h}, \quad \mu^{h}=\sum_{K} \mu_{K}^{h} B_{K}, \quad \mu_{K}^{h} \leq 0\right\} .
$$

Proof: Let $\mu^{h} \in M^{h}$. For all $\varphi^{h} \in W^{h}$, we have :

$$
\int_{\Gamma_{C}} \mu^{h} \varphi^{h} \mathrm{~d} \Gamma=\sum_{K} \int_{\Gamma_{C}} \mu_{K}^{h} B_{K} \varphi^{h} \mathrm{~d} \Gamma=\sum_{K} \mu_{K}^{h}\left(\Pi_{\lambda}^{h} \varphi^{h}\right)_{K} \int_{\Gamma_{C}} B_{K} \mathrm{~d} \Gamma .
$$

Let us remind that $\left(\Pi_{\lambda}^{h} \varphi^{h}\right)_{K}=\int_{\Gamma_{C}} \varphi^{h} B_{K} \mathrm{~d} \Gamma / \int_{\Gamma_{C}} B_{K} \mathrm{~d} \Gamma$ where $B_{K}$ are the basis functions of the multipliers on $\Gamma_{C}$.

For each $K, K^{\prime}$, we wish to construct a $\varphi_{K}^{h}$ such that

$$
\left(\Pi_{\lambda}^{h} \varphi_{K}^{h}\right)_{K^{\prime}}=\delta_{K, K^{\prime}}
$$

Clearly such $\varphi_{K}^{h}$, if it exists, belongs to $L^{h}$ by construction. Moreover, as $\mu^{h} \in M^{h}$,

$\int_{\Gamma_{C}} \mu^{h} \varphi_{K}^{h} \mathrm{~d} \Gamma=\mu_{K}^{h} \int_{\Gamma_{C}} B_{K} \mathrm{~d} \Gamma \leq 0$, i.e. $\mu_{K}^{h} \leq 0$.

Now, it remains to construct $\varphi_{K}^{h}$ verifying (17).

By definition of $\left(\Pi_{\lambda}^{h} \cdot\right)_{K}$, such a $\varphi_{K}^{h}$ has to verify

$$
\int_{\Gamma_{C}} \varphi_{K}^{h} B_{K^{\prime}} \mathrm{d} \Gamma=\delta_{K, K^{\prime}} \int_{\Gamma_{C}} B_{K^{\prime}} \mathrm{d} \Gamma
$$

and the existence of such $\varphi_{K}^{h}$ is guaranteed by the inf - sup condition. Indeed, it guaranties that the rectangular system (18) is solvable as the matrix is full rank.

Then a discretized mixed formulation of the problem (5) consists in finding $\left(u^{h}, \lambda^{h}\right) \in V^{h} \times M^{h}$ such that:

$$
\left\{\begin{aligned}
a\left(u^{h}, v^{h}\right)-b\left(\lambda^{h}, v^{h}\right)=L\left(v^{h}\right), & \forall v^{h} \in V^{h}, \\
b\left(\mu^{h}-\lambda^{h}, u^{h}\right) \geq 0, & \forall \mu^{h} \in M^{h} .
\end{aligned}\right.
$$

According to Lemma 2.3, we get:

$$
\left.\left\{\mu^{h} \in M^{h}: \quad b\left(\mu^{h}, v^{h}\right)=0 \quad \forall v^{h} \in V^{h}\right)\right\}=\{0\},
$$

and using the ellipticity of the bilinear form $a(\cdot, \cdot)$ on $V^{h}$, then the problem (19) admits a unique solution $\left(u^{h}, \lambda^{h}\right) \in V^{h} \times M^{h}$.

Before addressing the analysis of (19), let us recall that the following inequalities (see [2]) are true for the primal and the dual space.

Theorem 2.4. Let a given quasi-uniform mesh and let $r, s$ be such that $0 \leq r \leq s \leq p+1$. Then, there exists a constant depending only on $p, \theta, \varphi_{0}$ and $\hat{W}$ such that for any $v \in H^{s}(\Omega)$ there exists an approximation $v^{h} \in V^{h}$ such that

$$
\left\|v-v^{h}\right\|_{r, \Omega} \lesssim h^{s-r}\|v\|_{s, \Omega} .
$$


We will also make use of the local approximation estimates for splines quasi-interpolants that can be found e.g. in $[2,3]$.

Lemma 2.5. Let $\lambda \in H^{s}\left(\Gamma_{C}\right)$ with $0 \leq s \leq p-1$, then there exists a constant depending only on $p, \varphi_{0}$ and $\theta$, there exists an approximation $\lambda^{h} \in \Lambda^{h}$ such that:

$$
h^{-1 / 2}\left\|\lambda-\lambda^{h}\right\|_{-1 / 2, Q_{C}}+\left\|\lambda-\lambda^{h}\right\|_{0, Q_{C}} \lesssim h^{s}\|\lambda\|_{s, \tilde{Q}_{C}},\left.\quad \forall Q_{C} \in \mathcal{Q}_{h}\right|_{\Gamma_{C}} .
$$

It is well known [5] that the stability for the mixed problem (5) is linked to the inf - sup condition.

Theorem 2.6. For $h$ sufficiently small, $n$ sufficiently regular and for any $\mu^{h} \in \Lambda^{h}$, it holds:

$$
\sup _{v^{h} \in V^{h}} \frac{b\left(\mu^{h}, v^{h}\right)}{\left\|v^{h}\right\|_{V}} \geq \beta\left\|\mu^{h}\right\|_{W^{\prime}},
$$

where $\beta$ is independent of $h$.

Proof: By Proposition 2.2, there exists a Fortin's operator $\Pi:\left.L^{2}\left(\Gamma_{C}\right) \rightarrow V^{h}\right|_{\Gamma_{C}} \cap H_{0}^{1}\left(\Gamma_{C}\right)$ such that

$$
b(\lambda, \Pi(u))=b(\lambda, u), \forall \lambda \in M \quad \text { and } \quad\|\Pi(u)\|_{0, \Gamma_{C}} \leq\|u\|_{0, \Gamma_{C}} .
$$

Let $I_{h}$ be a $L^{2}$ and $H^{1}$ stable quasi-interpolant onto $\left.V^{h}\right|_{\Gamma_{C}}$ (for example, the Schumaker's quasiinterpolant, see for more details [3]). It is important to notice that $I^{h}$ preserves the homogeneous Dirichlet boundary condition.

We set $\Pi_{F}=\Pi\left(I-I_{h}\right)+I_{h}$. It is classical to see that:

$$
b\left(\lambda, \Pi_{F}(u)\right)=b(\lambda, u), \quad \forall \lambda \in M,
$$

and it is easy to see that:

$$
\Pi_{F}\left(u^{h}\right)=u^{h},\left.\quad \forall u^{h} \in V^{h}\right|_{\Gamma_{C}} .
$$

Moreover, by stability of $\Pi$ and $I_{h}$, it holds:

$$
\left\|\Pi_{F}(u)\right\|_{0, \Gamma_{C}} \lesssim\|u\|_{0, \Gamma_{C}}, \quad \forall u \in L^{2}\left(\Gamma_{C}\right) .
$$

and also

$$
\left\|\Pi_{F}(u)\right\|_{1, \Gamma_{C}} \lesssim\|(u)\|_{1, \Gamma_{C}}, \quad \forall u \in H^{1}\left(\Gamma_{C}\right) .
$$

To conclude, we distinguish between two cases :

- If $\bar{\Gamma}_{D} \cap \bar{\Gamma}_{C}=\emptyset$, it is well know that $W=H^{1 / 2}\left(\Gamma_{C}\right)$. By interpolation of Sobolev Spaces, using (25) and (26), we obtain:

$$
b\left(\lambda, \Pi_{F}(u)\right)=b(\lambda, u), \quad \forall \lambda \in M \quad \text { and } \quad\left\|\Pi_{F}(u)\right\|_{W} \lesssim\|u\|_{W} .
$$

Then inf - sup condition (22) holds thanks to Proposition 5.4.2 of [5].

- If $\bar{\Gamma}_{D} \cap \bar{\Gamma}_{C} \neq \emptyset$, it is enough to remind that for all $u \in H_{0, \Gamma_{D} \cap \Gamma_{C}}^{1}\left(\Gamma_{C}\right)$, we have $\Pi_{F}(u) \in$ $H_{0, \Gamma_{D} \cap \Gamma_{C}}^{1}\left(\Gamma_{C}\right)$ and $(26)$ is valid on the subspace $H_{0, \Gamma_{D} \cap \Gamma_{C}}^{1}\left(\Gamma_{C}\right)$. Again by interpolation argument between (25) and (26), it holds $\left\|\Pi_{F}(u)\right\|_{W} \leq C\|u\|_{W}$ which ends the proof. 


\section{A priori error analysis}

In this section, we present an optimal a priori error estimate for the Signorini mixed problem. Our estimates follows the ones for finite elements, provided in [7, 14], and refined in [11]. In particular, in [11] the authors overcome a technical assumption on the geometric structure of the contact set and we are able to avoid such as assumptions also in our case.

Indeed, for any $p$, we prove our method to be optimal for solutions with regularity up to $5 / 2$. Thus, optimality for the displacement is obtained for any $p \geq 2$. The cheapest and more convenient method proved optimal corresponds to the choice $p=2$. Larger values of $p$ may be of interest because they produce continuous pressures, but, on the other hand, the error bounds remain limited by the regularity of the solution, i.e. , up to $C h^{3 / 2}$. Clearly, to enhance approximation suitable local refinement may be used, [10], but this choice outside the scope of this paper.

In order to prove Theorem 3.3 which follows, we need a few preparatory Lemmas.

First, we introduce some notation and some basic estimates. Let us define the active-set strategy

for the variational problem. Given an element $\left.Q_{C} \in \mathcal{Q}_{h}\right|_{\Gamma_{C}}$ of the undeformed mesh, we denote by $Z_{C}\left(Q_{C}\right)$ the contact set and by $Z_{N C}\left(Q_{C}\right)$ the non-contact set in $Q_{C}$, as follows:

$$
Z_{C}\left(Q_{C}\right)=\left\{x \in Q_{C}, \quad u_{n}(x)=0\right\} \quad \text { and } \quad Z_{N C}\left(Q_{C}\right)=\left\{x \in Q_{C}, \quad u_{n}(x)>0\right\} .
$$

$\left|Z_{C}\left(Q_{C}\right)\right|$ and $\left|Z_{N C}\left(Q_{C}\right)\right|$ stand for their measures and $\left|Z_{C}\left(Q_{C}\right)\right|+\left|Z_{N C}\left(Q_{C}\right)\right|=\left|Q_{C}\right|=C h_{Q_{C}}^{d-1}$.

Remark 3.1. Since $u_{n}$ belongs to $H^{1+\nu}(\Omega)^{2}$ for $0<\nu<1$, if $d=2$ the Sobolev embeddings ensure that $u_{n} \in \mathcal{C}^{0}(\partial \Omega)$. It implies that $Z_{C}\left(Q_{C}\right)$ and $Z_{N C}\left(Q_{C}\right)$ are measurable as inverse images of a set by a continuous function.

The following estimates are the generalization to the mixed problem of Lemma 2 of Appendix of the article [11]. We recall that if $(u, \lambda)$ is a solution of the mixed problem $(5)$ then $\sigma_{n}(u)=\lambda$. So, the following lemma can be proven exactly in the same way.

Lemma 3.2. Let $d=2$ or 3 . Let $(u, \lambda)$ be the solution of the mixed formulation (5) and let $u \in H^{3 / 2+\nu}(\Omega)^{d}$ with $0<\nu<1$. Let $h_{Q}$ the be the diameter of the trace element $Q_{C}$ and the set of contact $Z_{C}\left(Q_{C}\right)$ and non-contact $Z_{N C}\left(Q_{C}\right)$ defined previously in $Q_{C}$.

We assume that $\left|Z_{N C}\left(Q_{C}\right)\right|>0$, the following $L^{2}$-estimate holds for $\lambda$ :

$$
\|\lambda\|_{0, Q_{C}} \leq \frac{1}{\left|Z_{N C}\left(Q_{C}\right)\right|^{1 / 2}} h_{Q_{C}}^{d / 2+\nu-1 / 2}|\lambda|_{\nu, Q_{C}} .
$$

We assume that $\left|Z_{C}\left(Q_{C}\right)\right|>0$, the following $L^{2}$-estimates hold for $\nabla u_{n}$ :

$$
\left\|\nabla u_{n}\right\|_{0, Q_{C}} \leq \frac{1}{\left|Z_{C}\left(Q_{C}\right)\right|^{1 / 2}} h_{Q_{C}}^{d / 2+\nu-1 / 2}\left|\nabla u_{n}\right|_{\nu, Q_{C}} .
$$

Theorem 3.3. Let $(u, \lambda)$ and $\left(u^{h}, \lambda^{h}\right)$ be respectively the solution of the mixed problem (5) and the discrete mixed problem (19). Assume that $u \in H^{3 / 2+\nu}(\Omega)^{d}$ with $0<\nu<1$. Then, the following error estimate is satisfied:

$$
\left\|u-u^{h}\right\|_{V}^{2}+\left\|\lambda-\lambda^{h}\right\|_{W^{\prime}}^{2} \lesssim h^{1+2 \nu}\|u\|_{3 / 2+\nu, \Omega}^{2}
$$


Proof: In the article [14] Proposition 4.1, it is proved that if $(u, \lambda)$ is the solution of the mixed problem (5) and $\left(u^{h}, \lambda^{h}\right)$ is the solution of the discrete mixed problem (19), it holds :

$$
\begin{aligned}
\left\|u-u^{h}\right\|_{V}^{2}+\left\|\lambda-\lambda^{h}\right\|_{W^{\prime}}^{2} \lesssim & \left\|u-v^{h}\right\|_{V}^{2}+\left\|\lambda-\mu^{h}\right\|_{W^{\prime}}^{2} \\
& +\max \left(-b\left(\lambda, u^{h}\right), 0\right)+\max \left(-b\left(\lambda^{h}, u\right), 0\right) .
\end{aligned}
$$

It remains to estimate on the previous inequality the last two terms to obtain the estimate (29).

Step 1: estimate of $-b\left(\lambda, u^{h}\right)=\int_{\Gamma_{C}} \lambda u_{n}^{h} \mathrm{~d} \Gamma$.

Using the operator $\Pi_{\lambda}^{h}$ defined in (9), it holds:

$$
\begin{aligned}
-b\left(\lambda, u^{h}\right)= & \int_{\Gamma_{C}} \lambda u_{n}^{h} \mathrm{~d} \Gamma=\int_{\Gamma_{C}} \lambda\left(u_{n}^{h}-\Pi_{\lambda}^{h}\left(u_{n}^{h}\right)\right) \mathrm{d} \Gamma+\int_{\Gamma_{C}} \lambda \Pi_{\lambda}^{h}\left(u_{n}^{h}\right) \mathrm{d} \Gamma \\
= & \int_{\Gamma_{C}}\left(\lambda-\Pi_{\lambda}^{h}(\lambda)\right)\left(u_{n}^{h}-\Pi_{\lambda}^{h}\left(u_{n}^{h}\right)\right) \mathrm{d} \Gamma+\int_{\Gamma_{C}} \Pi_{\lambda}^{h}(\lambda)\left(u_{n}^{h}-\Pi_{\lambda}^{h}\left(u_{n}^{h}\right)\right) \mathrm{d} \Gamma \\
& +\int_{\Gamma_{C}} \lambda \Pi_{\lambda}^{h}\left(u_{n}^{h}\right) \mathrm{d} \Gamma .
\end{aligned}
$$

Since $\lambda$ is a solution of $(5)$, it holds $\Pi_{\lambda}^{h}(\lambda) \leq 0$. Furthermore, $u^{h}$ is a solution of (19), thus $\int_{\Gamma_{C}} \Pi_{\lambda}^{h}(\lambda)\left(u_{n}^{h}-\Pi_{\lambda}^{h}\left(u_{n}^{h}\right)\right) \mathrm{d} \Gamma \leq 0$ and $\int_{\Gamma_{C}} \lambda \Pi_{\lambda}^{h}\left(u_{n}^{h}\right) \mathrm{d} \Gamma \leq 0$.

We obtain:

$$
\begin{aligned}
-b\left(\lambda, u^{h}\right) \leq & \int_{\Gamma_{C}}\left(\lambda-\Pi_{\lambda}^{h}(\lambda)\right)\left(u_{n}^{h}-\Pi_{\lambda}^{h}\left(u_{n}^{h}\right)\right) \mathrm{d} \Gamma \\
\leq & \int_{\Gamma_{C}}\left(\lambda-\Pi_{\lambda}^{h}(\lambda)\right)\left(u_{n}^{h}-u_{n}-\Pi_{\lambda}^{h}\left(u_{n}^{h}-u_{n}\right)\right) \mathrm{d} \Gamma \\
& +\int_{\Gamma_{C}}\left(\lambda-\Pi_{\lambda}^{h}(\lambda)\right)\left(u_{n}-\Pi_{\lambda}^{h}\left(u_{n}\right)\right) \mathrm{d} \Gamma .
\end{aligned}
$$

The first term of (30) is bounded in an optimal way by using (10), the summation on each physical element, Theorem 1.1 and the trace theorem:

$$
\begin{aligned}
\int_{\Gamma_{C}}\left(\lambda-\Pi_{\lambda}^{h}(\lambda)\right)\left(u_{n}^{h}-u_{n}-\Pi_{\lambda}^{h}\left(u_{n}^{h}-u_{n}\right)\right) \mathrm{d} \Gamma & \leq\left\|\lambda-\Pi_{\lambda}^{h}(\lambda)\right\|_{0, \Gamma_{C}}\left\|u_{n}^{h}-u_{n}-\Pi_{\lambda}^{h}\left(u_{n}^{h}-u_{n}\right)\right\|_{0, \Gamma_{C}} \\
& \leq C h^{1 / 2+\nu}\|\lambda\|_{\nu, \Gamma_{C}}\left\|u_{n}-u_{n}^{h}\right\|_{W} \\
& \leq C h^{1 / 2+\nu}\|u\|_{3 / 2+\nu, \Omega}\left\|u-u^{h}\right\|_{V} .
\end{aligned}
$$

We need now to bound the second term in (30). Let $Q_{C}$ be an element of $\left.\mathcal{Q}_{h}\right|_{\Gamma_{C}}$. If either $\left|Z_{C}\left(Q_{C}\right)\right|$ or $\left|Z_{N C}\left(Q_{C}\right)\right|$ are null, the integral on $Q_{C}$ vanishes. So we suppose that either $\left|Z_{C}\left(Q_{C}\right)\right|$ or $\left|Z_{N C}\left(Q_{C}\right)\right|$ are greater than $\left|Q_{C}\right| / 2=C h_{Q_{C}}^{d-1}$ and we consider the two cases, separately.

Similarly to the article [14], we can prove that if: 
- $\left|Z_{C}\left(Q_{C}\right)\right| \geq\left|Q_{C}\right| / 2$. Using the estimate (10), the estimate (28) of Lemma 3.2 and the Young's inequality, it holds:

$$
\int_{Q_{C}}\left(\lambda-\Pi_{\lambda}^{h}(\lambda)\right)\left(u_{n}-\Pi_{\lambda}^{h}\left(u_{n}\right)\right) \mathrm{d} \Gamma \lesssim h^{1+2 \nu}\left(\|\lambda\|_{\nu, Q_{C}}^{2}+\left\|u_{n}\right\|_{1+\nu, \tilde{Q}_{C}}^{2}\right) .
$$

- $\left|Z_{N C}\left(Q_{C}\right)\right| \geq\left|Q_{C}\right| / 2$. Using the estimate (10), the estimate (27) of Lemma 3.2 and the Young's inequality, it holds:

$$
\int_{Q_{C}}\left(\lambda-\Pi_{\lambda}^{h}(\lambda)\right)\left(u_{n}-\Pi_{\lambda}^{h}\left(u_{n}\right)\right) \mathrm{d} \Gamma \lesssim h^{1+2 \nu}\left(\|\lambda\|_{\nu, \tilde{Q}_{C}}^{2}+\left\|u_{n}\right\|_{1+\nu, \tilde{Q}_{C}}^{2}\right)
$$

Summing over all the contact elements and distinguishing the two cases $Z_{C}\left(Q_{C}\right) \geq\left|Q_{C}\right| / 2$ and $Z_{N C}\left(Q_{C}\right) \geq\left|Q_{C}\right| / 2$, it holds:

$$
\begin{aligned}
\int_{\Gamma_{C}}\left(\lambda-\Pi_{\lambda}^{h}(\lambda)\right)\left(u_{n}-\Pi_{\lambda}^{h}\left(u_{n}\right)\right) \mathrm{d} \Gamma & =\sum_{\left.Q_{C} \in \mathcal{Q}_{h}\right|_{\Gamma_{C}}} \int_{Q_{C}}\left(\lambda-\Pi_{\lambda}^{h}(\lambda)\right)\left(u_{n}-\Pi_{\lambda}^{h}\left(u_{n}\right)\right) \mathrm{d} \Gamma \\
& \leq C h^{1+2 \nu} \sum_{\left.Q_{C} \in \mathcal{Q}_{h}\right|_{\Gamma_{C}}}\|\lambda\|_{\nu, Q_{C}}^{2}+\|\lambda\|_{\nu, \tilde{Q}_{C}}^{2}+\left\|u_{n}\right\|_{1+\nu, \tilde{Q}_{C}}^{2} \\
& \leq C h^{1+2 \nu} \sum_{\left.Q_{C} \in \mathcal{Q}_{h}\right|_{\Gamma_{C}}}\|\lambda\|_{\nu, Q_{C}}^{2}+\sum_{Q_{C}^{\prime} \in \tilde{Q}_{C}}\|\lambda\|_{\nu, Q_{C}^{\prime}}^{2}+\left\|u_{n}\right\|_{1+\nu, Q_{C}^{\prime}}^{2} \\
& \leq C h^{1+2 \nu}\left(\|\lambda\|_{\nu, \Gamma_{C}}^{2}+\sum_{\left.Q \in \mathcal{Q}_{h}\right|_{\Gamma_{C}}} \sum_{Q_{C}^{\prime} \in \tilde{Q}_{C}}\|\lambda\|_{\nu, Q_{C}^{\prime}}^{2}+\left\|u_{n}\right\|_{1+\nu, Q_{C}^{\prime}}^{2}\right) .
\end{aligned}
$$

Due to the compact supports of the B-Splines basis functions, there exists a constant $C$ depending only on the degree $p$ and the dimension $d$ of the undeformed domain such that:

$$
\sum_{\left.Q \in \mathcal{Q}_{h}\right|_{\Gamma_{C}}} \sum_{Q_{C}^{\prime} \in \tilde{Q}_{C}}\|\lambda\|_{\nu, Q_{C}^{\prime}}^{2}+\left\|u_{n}\right\|_{1+\nu, Q_{C}^{\prime}}^{2} \leq C\|\lambda\|_{\nu, \Gamma_{C}}^{2}+C\left\|u_{n}\right\|_{1+\nu, \Gamma_{C}}^{2} .
$$

So we have:

$$
\int_{\Gamma_{C}}\left(\lambda-\Pi_{\lambda}^{h}(\lambda)\right)\left(u_{n}-\Pi_{\lambda}^{h}\left(u_{n}\right)\right) \mathrm{d} \Gamma \leq C h^{1+2 \nu}\left(\|\lambda\|_{\nu, \Gamma_{C}}^{2}+\left\|u_{n}\right\|_{1+\nu, \Gamma_{C}}^{2}\right),
$$

i.e.

$$
\int_{\Gamma_{C}}\left(\lambda-\Pi_{\lambda}^{h}(\lambda)\right)\left(u_{n}-\Pi_{\lambda}^{h}\left(u_{n}\right)\right) \mathrm{d} \Gamma \leq C h^{1+2 \nu}\|u\|_{3 / 2+\nu, \Omega}^{2} .
$$

We conclude that:

$$
-b\left(\lambda, u^{h}\right) \lesssim h^{1 / 2+\nu}\|u\|_{3 / 2+\nu, \Omega}\left\|u-u^{h}\right\|_{V}+h^{1+2 \nu}\|u\|_{3 / 2+\nu, \Omega}^{2} .
$$

Using Young's inequality, we obtain:

$$
-b\left(\lambda, u^{h}\right) \lesssim h^{1+2 \nu}\|u\|_{3 / 2+\nu, \Omega}^{2}+\left\|u-u^{h}\right\|_{V}^{2} .
$$


Step 2: estimate of $-b\left(\lambda^{h}, u\right)=\int_{\Gamma_{C}} \lambda^{h} u_{n} \mathrm{~d} \Gamma$.

Let us denote by $j^{h}$ the Lagrange interpolation operator of order one on $\left.\mathcal{Q}_{h}\right|_{\Gamma_{C}}$.

$$
-b\left(\lambda^{h}, u\right)=\int_{\Gamma_{C}} \lambda^{h} u_{n} \mathrm{~d} \Gamma=\int_{\Gamma_{C}} \lambda^{h}\left(u_{n}-j^{h}\left(u_{n}\right)\right) \mathrm{d} \Gamma+\int_{\Gamma_{C}} \lambda^{h} j^{h}\left(u_{n}\right) \mathrm{d} \Gamma .
$$

Note that by remark $3.1, u_{n}$ is continuous and $j^{h}\left(u_{n}\right)$ is well define.

Since $u$ is a solution of $(5)$, it holds $j^{h}\left(u_{n}\right) \geq 0$. Thus, $\int_{\Gamma_{C}} \lambda^{h} j^{h}\left(u_{n}\right) \mathrm{d} \Gamma \leq 0, \quad \lambda^{h} \in M^{h}$.

As previously, we obtain:

$$
\begin{aligned}
-b\left(\lambda^{h}, u\right) & \leq \int_{\Gamma_{C}} \lambda^{h} u_{n} \mathrm{~d} \Gamma \leq \int_{\Gamma_{C}} \lambda^{h}\left(u_{n}-j^{h}\left(u_{n}\right)\right) \mathrm{d} \Gamma \\
& \leq \int_{\Gamma_{C}}\left(\lambda^{h}-\lambda\right)\left(u_{n}-j^{h}\left(u_{n}\right)\right) \mathrm{d} \Gamma+\int_{\Gamma_{C}} \lambda\left(u_{n}-j^{h}\left(u_{n}\right)\right) \mathrm{d} \Gamma \\
& \leq \int_{\Gamma_{C}}^{\lambda} \lambda\left(u_{n}-j^{h}\left(u_{n}\right)\right) \mathrm{d} \Gamma+\left\|\lambda-\lambda^{h}\right\|_{W^{\prime}}\left\|u_{n}-j^{h}\left(u_{n}\right)\right\|_{W} \\
& \leq \int_{\Gamma_{C}} \lambda\left(u_{n}-j^{h}\left(u_{n}\right)\right) \mathrm{d} \Gamma+h^{1 / 2+\nu}\left\|u_{n}\right\|_{1+\nu, \Gamma_{C}}\left\|\lambda-\lambda^{h}\right\|_{W^{\prime}} \\
& \leq \int_{\Gamma_{C}}^{\lambda} \lambda\left(u_{n}-j^{h}\left(u_{n}\right)\right) \mathrm{d} \Gamma+h^{1 / 2+\nu}\|u\|_{3 / 2+\nu, \Omega}\left\|\lambda-\lambda^{h}\right\|_{W^{\prime}} .
\end{aligned}
$$

Now, we need to show that:

$$
\int_{\Gamma_{C}} \lambda\left(u_{n}-j^{h}\left(u_{n}\right)\right) \mathrm{d} \Gamma \leq C h^{1+2 \nu}\|u\|_{3 / 2+\nu, \Omega}^{2}
$$

The proof of this inequality is done in the paper [11] for both linear and quadratic finite elements, and can be repeated here verbatim. In this proof, two cases are considered:

1. either $\left|Z_{C}\left(Q_{C}\right)\right|$ or $\left|Z_{N C}\left(Q_{C}\right)\right|$ is null and thus the inequality is trivial;

2. where either $\left|Z_{C}\left(Q_{C}\right)\right|$ or $\left|Z_{N C}\left(Q_{C}\right)\right|$ is greater than $\left|Q_{C}\right| / 2=C h_{Q_{C}}^{d-1}$.

As previously, choosing either $\left|Z_{C}\left(Q_{C}\right)\right|$ or $\left|Z_{N C}\left(Q_{C}\right)\right|$, using the previous Lemma 3.2 and by summation on all element of mesh, we conclude that:

$$
\begin{aligned}
-b\left(\lambda^{h}, u\right) & \leq \int_{\Gamma_{C}} \lambda\left(u_{n}-j^{h}\left(u_{n}\right)\right) \mathrm{d} \Gamma+h^{1 / 2+\nu}\|u\|_{3 / 2+\nu, \Omega}\left\|\lambda-\lambda^{h}\right\|_{W^{\prime}} \\
& \lesssim h^{1+2 \nu}\|u\|_{3 / 2+\nu, \Omega}^{2}+h^{1 / 2+\nu}\|u\|_{3 / 2+\nu, \Omega}\left\|\lambda-\lambda^{h}\right\|_{W^{\prime}} .
\end{aligned}
$$

Using Young's inequality, we obtain:

$$
-b\left(\lambda^{h}, u\right) \lesssim h^{1+2 \nu}\|u\|_{3 / 2+\nu, \Omega}^{2}+\left\|\lambda-\lambda^{h}\right\|_{W^{\prime}}^{2} .
$$

Finally, we can conclude using (33) and (31). 


\section{Numerical Study}

In this section, we perform a numerical validation for the method we propose $\mathrm{d}$ in small as well as in large deformation frameworks, i.e., also beyond the theory developed in previous Sections. Due to the intrinsic lack of regularity of contact solutions, we restrict ourselves to the case $p=2$, for which the $N_{2} / S_{0}$ method is tested.

The suite of benchmarks reproduces the classical Hertz contact problem [13]: Sections 4.1 and 4.1 analyse the two and three-dimensional cases for a small deformation setting, whereas Section 4.3 considers the large deformation problem in 2D. The examples were performed using an in-house code based on the igatools library (see [21] for further details).

In the following example, to prevent that the contact zone is empty, we considered, only for the initial gap, that there exists contact if the $g_{n} \leq 10^{-9}$.

\subsection{Two-dimensional Hertz problem}

The example included in this section analyses the two-dimensional frictionless Hertz contact problem considering small elastic deformations. It consists in an infinitely long half cylinder body with radius $R=1$, that it is deformable and whose material is linear elastic, with Young's modulus $E=1$ and Poisson's ratio $\nu=0.3$. A uniform pressure $P=0.003$ is applied on the top face of the cylinder while the curved surface contacts against a horizontal rigid plane (see Figure 1(a)). Taking into account the test symmetry and the ideally infinite length of the cylinder, the problem is modelled as 2D quarter of disc with proper boundary conditions.

Under the hypothesis that the contact area is small compared to the cylinder dimensions, the Hertz's analytical solution (see [13]) predicts that the contact region is an infinitely long band whose width is $2 a$, being $a=\sqrt{8 R^{2} P\left(1-\nu^{2}\right) / \pi E}$. Thus, the normal pressure, that follows an elliptical distribution along the width direction $r$, is $p(r)=p_{0} \sqrt{1-r^{2} / a^{2}}$, where the maximum pressure, at the central line of the band $(r=0)$, is $p_{0}=4 R P / \pi a$. For the geometrical, material and load data chosen in this numerical test, the characteristic values of the solution are $a=0.083378$ and $p_{0}=0.045812$. Notice that, as required by Hertz's theory hypotheses, $a$ is sufficiently small compared to $R$.

It is important to remark that, despite the fact that Hertz's theory provides a full description of the contact area and the normal contact pressure in the region, it does not describe analytically the deformation of the whole elastic domain. Therefore, for all the test cases hereinafter, the $L^{2}$ error norm and $H^{1}$ error semi-norm of the displacement obtained numerically are computed taking a more refined solution as a reference. For this bidimensional test case, the mesh size of the refined solution $h_{r e f}$ is such that, for all the discretizations, $4 h_{r e f} \leq h$, where $h$ is the size of the mesh considered. Additionally, as it is shown in Figure 1(a), the mesh is finer in the vicinity of the potential contact zone. The knot vector values are defined such that $80 \%$ of the knot spans are located within $10 \%$ of the total length of the knot vector.

In particular, the analysis of this example focuses on the effect of the interpolation order on the quality of contact stress distribution. Thus, in Figure 1(b) we compare the pressure reference solution. with the Lagrange multiplier values computed at the control points, i.e. its constant values, and a post-processing which consists in a $P 1$ re-interpolation. The dimensionless contact pressure $p / p_{0}$ is plotted respect to the normalized coordinate $r / a$. The results are very good: the maximum pressure computed and the pressure distribution, even across the boundary of the contact region (on the contact and non contact zones), are close to the analytical solution. 


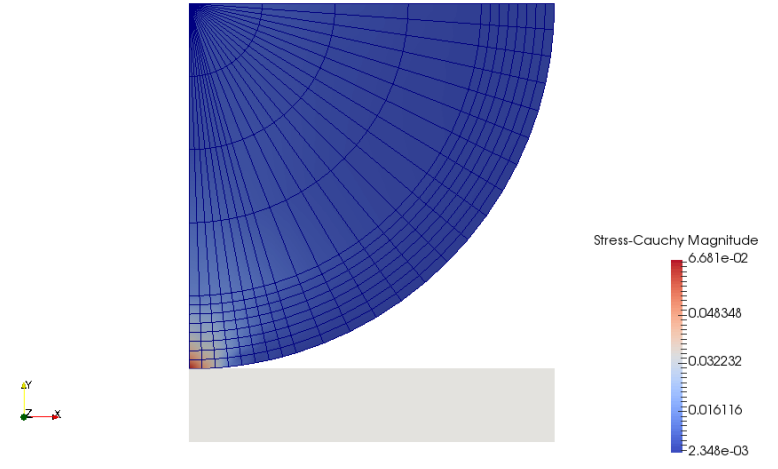

(a) Stress magnitude distribution for the undeformed mesh.

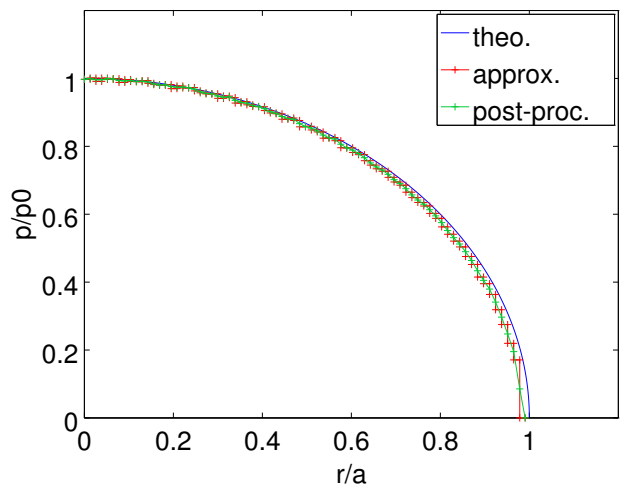

(b) Analytical and numerical contact pressure.

Figure 1: 2D Hertz contact problem with $N_{2} / S_{0}$ method for an applied pressure $P=0.003$.

In Figure 2(a), absolute errors in $L^{2}$-norm and $H^{1}$-semi-norm for the $N_{2} / S_{0}$ choice are shown. As expected, optimal convergence is obtained for the displacement error in the $H^{1}$-semi-norm: the convergence rate is close to the expected $3 / 2$ value. Nevertheless, the $L^{2}$-norm of the displacement error presents suboptimal convergence (close to 2), but according to Aubin-Nitsche's lemma in the linear case, the expected convergence rate is lower than $5 / 2$. On the other hand, in Figure 2(b) the $L^{2}$-norm of the Lagrange multipliers error is presented, the expected convergence rate is 1. Whereas a convergence rate close to 0.6 is achieved when we compare the numerical solution and the Hertz's analytical solution, and close to 0.8 is achieved when we compare the numerical solution and the refined numerical solution.

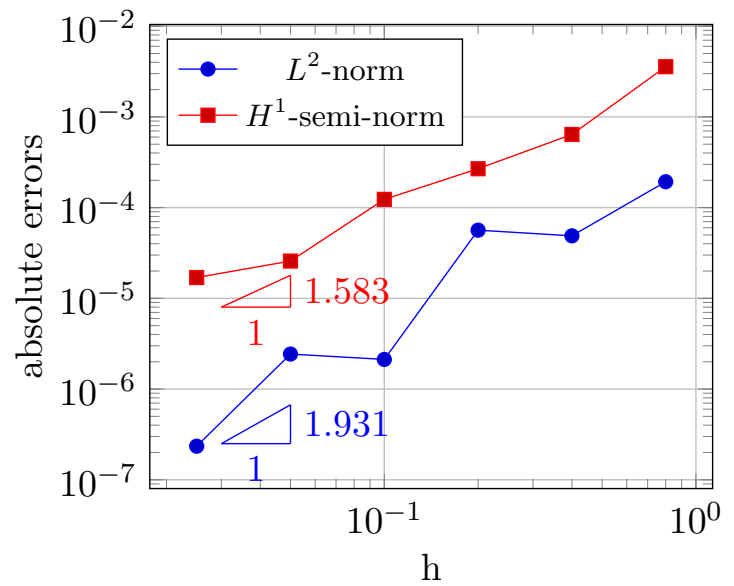

(a) Displacement error.

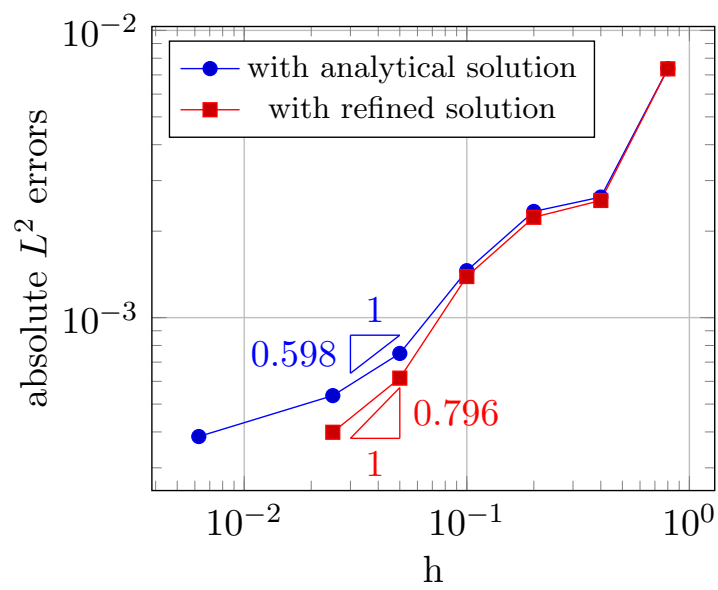

(b) Lagrange multipliers error.

Figure 2: 2D Hertz contact problem with $N_{2} / S_{0}$ method for an applied pressure $P=0.003$. Absolute displacement errors in $L^{2}$-norm and $H^{1}$-semi-norm and Lagrange multipliers error in $L^{2}$-norm, respect to analytical and refined numerical solutions. 


\subsection{Three-dimensional Hertz problem}

In this section, the three-dimensional frictionless Hertz problem is studied. It consists in a hemispherical elastic body with radius $R$ that contacts against a horizontal rigid plane as a consequence of an uniform pressure $P$ applied on the top face (see Figure 3(a)). Hertz's theory predicts that the contact region is a circle of radius $a=\left(3 R^{3} P\left(1-\nu^{2}\right) / 4 E\right)^{1 / 3}$ and the contact pressure follows a hemispherical distribution $p(r)=p_{0} \sqrt{1-r^{2} / a^{2}}$, with $p_{0}=3 R^{2} P / 2 a^{2}$, being $r$ the distance to the centre of the circle (see[13]). In this case, for the chosen values $R=1$, $E=1, \nu=0.3$ and $P=10^{-4}$, the contact radius is $a=0.059853$ and the maximum pressure $p_{0}=0.041872$. As in the two-dimensional case, Hertz's theory relies on the hypothesis that $a$ is small compared to $R$ and the deformations are small.

Considering the problem axial symmetry, the test is reproduced using an octant of sphere with proper boundary conditions. Figure 3(a) shows the problem setup and the magnitude of the computed stresses. As in the $2 \mathrm{D}$ case, in order to achieve more accurate results in the contact region, the mesh is refined in the vicinity of the potential contact zone. The knot vectors are defined such as $75 \%$ of the elements are located within $10 \%$ of the total length of the knot vector.

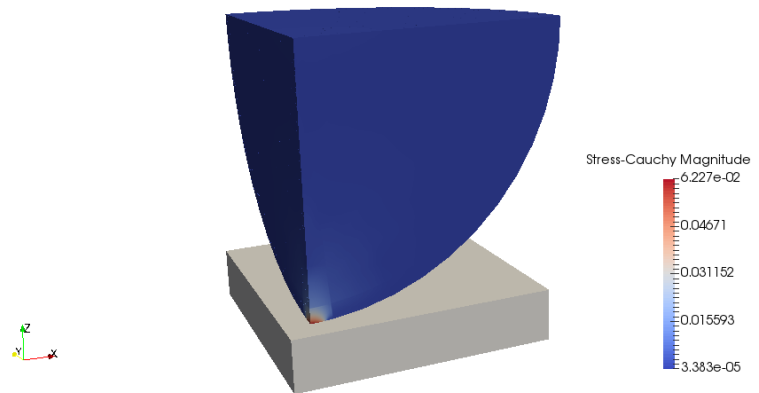

(a) magnitude for the undeformed mesh.

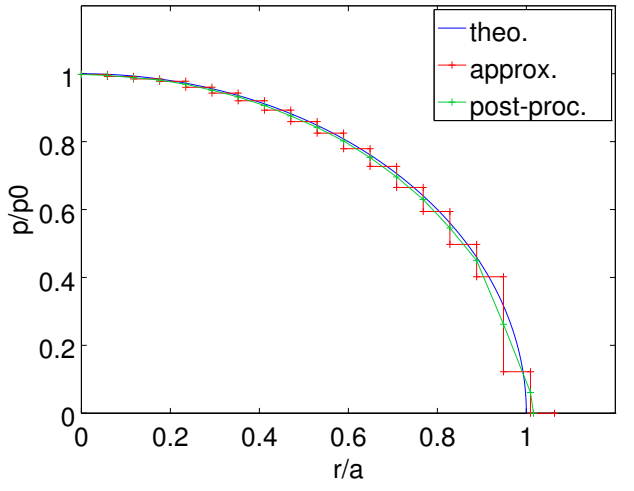

(b) Analytical and numerical contact pressure for $h=0.1$.

Figure 3: 3D Hertz contact problem with $N_{2} / S_{0}$ method for an applied pressure $P=10^{-4}$.

In Figure 3(b), we compare the Hertz's solution with the computed contact pressure at control points and a $P 1$ re-interpolation of those values, for a mesh with size $h=0.1$. On the other hand, in Figure 4 the contact pressure is shown at control points for mesh sizes $h=0.4$ and $h=0.2$. As it can be appreciated, good agreement between the analytical and computed pressure is obtained in all cases.

As in the previous test, the displacement of the deformed elastic body is not fully described by the Hertz's theory. Therefore, the $L^{2}$ error norm and $H^{1}$ error semi-norm of the displacement are evaluated by comparing the obtained solution with a finer refined case. Nonetheless, Lagrange multipliers computed solutions are compared with the analytical contact pressure. In this test case, the size of the refined mesh is $h_{r e f}=0.1175$ ( 0.0025 in the contact region), and it is such as $2 h_{\text {ref }} \leq h$.

In Figure 5(a) the displacement error norms are reported. As it can be seen, they present suboptimal convergence rates both in the $L^{2}$-norm and $H^{1}$-semi-norm. Convergence rates are close to 1.26 and 0.5 , respectively. The large mesh size of the numerical reference solution $h_{r e f}$, 


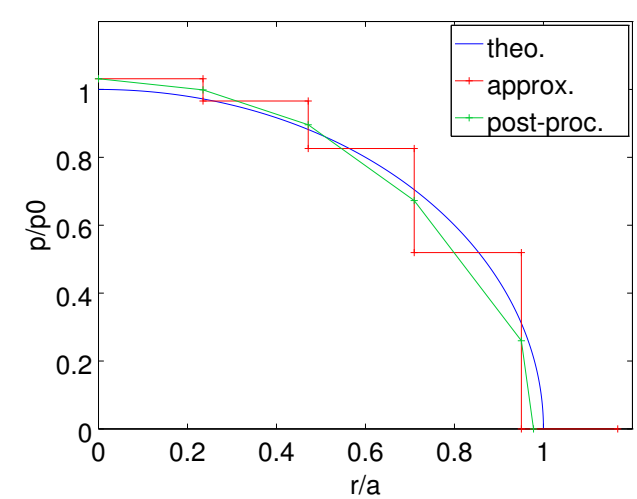

(a) $h=0.4$.

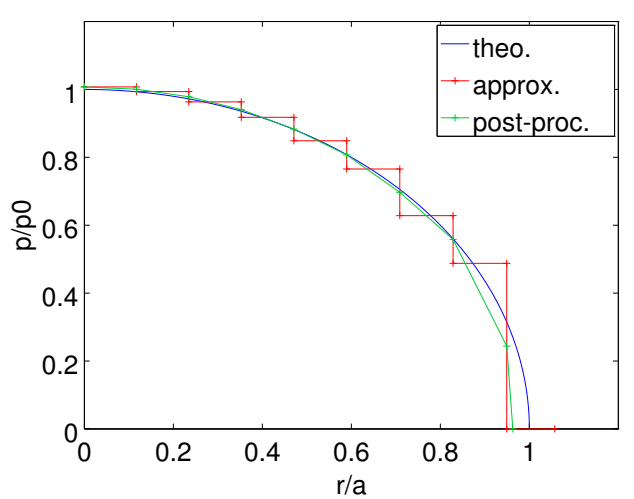

(b) $h=0.2$.

Figure 4: 3D Hertz contact problem with $N_{2} / S_{0}$ method for an applied pressure $P=10^{-4}$. Contact pressure solution at control points.

limited by our computational resources, seems to be the cause of these suboptimal results. Due to the coarse reference mesh, the presented rates are only pre-asymptotic. Better behaviour is observed for the Lagrange multipliers error (Figure 5(b)).

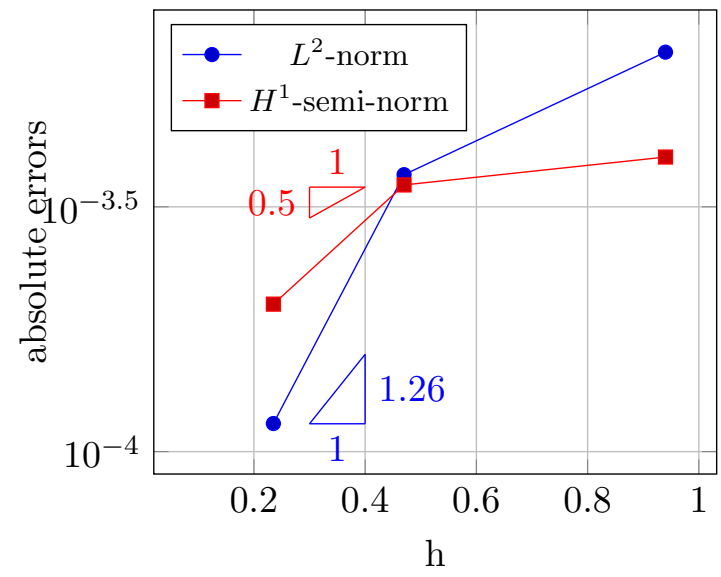

(a) Displacement error.

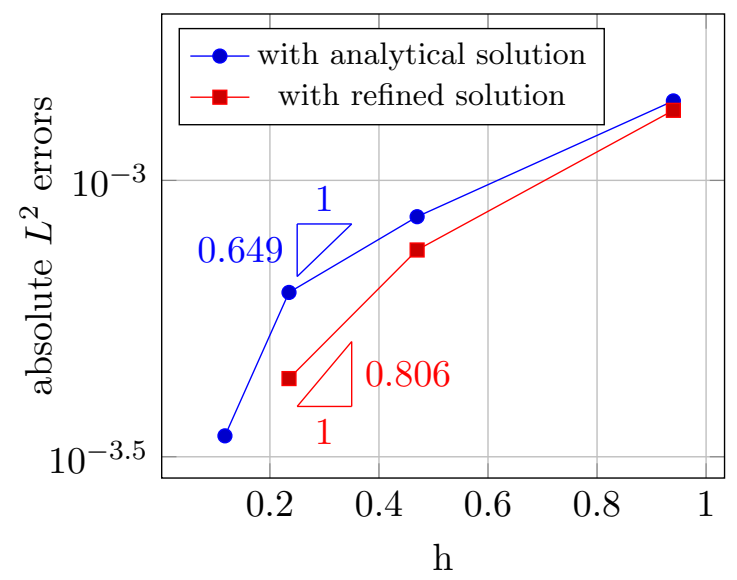

(b) Lagrange multipliers error.

Figure 5: 3D Hertz contact problem with $N_{2} / S_{0}$ method for an applied pressure $P=10^{-4}$. Absolute displacement errors in $L^{2}$-norm and $H^{1}$-semi-norm and Lagrange multipliers error in $L^{2}$-norm, respect to analytical and refined numerical solutions.

\subsection{Two-dimensional Hertz problem with large deformations}

Finally, in this section the two-dimensional frictionless Hertz problem is studied considering large deformations and strains. For that purpose, a Neo-Hookean material constitutive law (an hyperelastic law that considers finite strains) with Young's modulus $E=1$ and Poisson's ratio $\nu=0.3$, has been used for the deformable body.

As in Section 4.1, the performance of the $N_{2} / S_{0}$ method is analysed and the problem is mod- 
elled as an elastic quarter of disc with proper boundary conditions. The considerations made about the mesh size in Section 4.1 are also valid for the present case. The radius of the cylinder is $R=1$ but modifying its boundary conditions: instead of pressure, a uniform downward displacement $u_{y}=-0.4$ is applied on the top surface of the cylinder. In this large deformation framework the exact solution is unknown: the error of the computed displacement and Lagrange multipliers are studied taking a refined numerical solution as reference. The large deformation of the body and computed contact pressure are presented in Figure 6. In Figure 7, the displacement

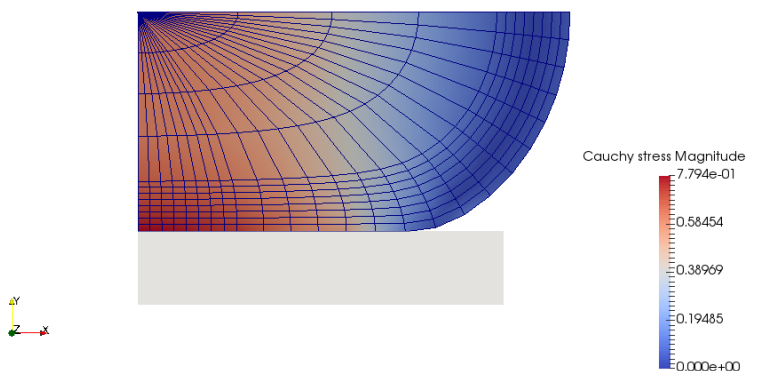

(a) Stress magnitude distribution for the deformed mesh.

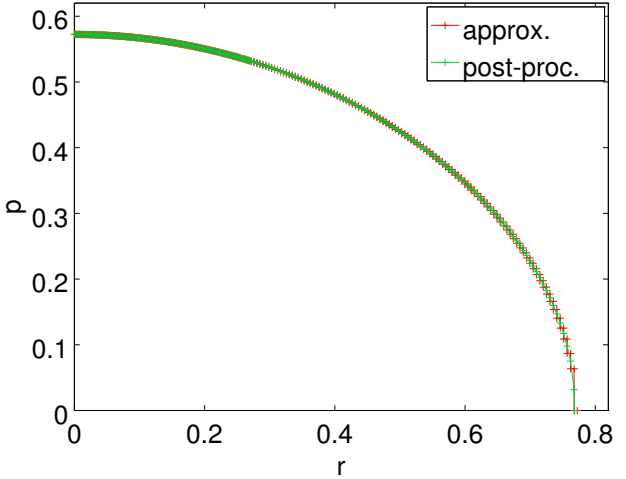

(b) Analytical and numerical contact pressure for $h=0.1$

Figure 6: 2D large deformation Hertz contact problem with $N_{2} / S_{0}$ method with a uniform downward displacement $u_{y}=-0.4$.

and multiplier errors are reported. It can be seen that the obtained displacement presents optimal convergence both in $L^{2}$-norm and $H^{1}$-semi-norm; analogously, optimal convergence is also achieved for the computed Lagrange multipliers.

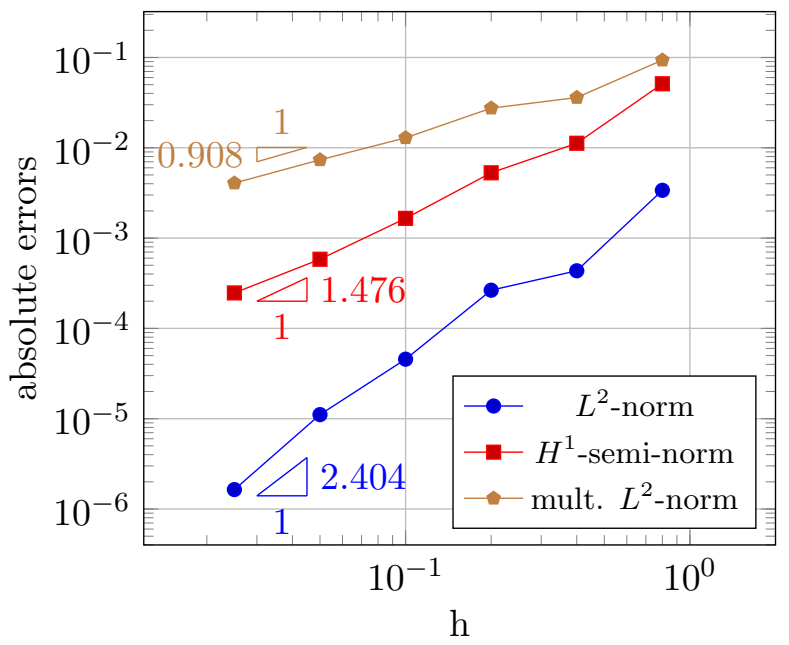

Figure 7: 2D large deformation Hertz contact problem with $N_{2} / S_{0}$ method with a uniform downward displacement $u_{y}=-0.4$. Absolute displacement errors in $L^{2}$-norm and $H^{1}$-semi-norm and Lagrange multipliers error in $L^{2}$-norm. 


\section{Conclusions}

In this work, we present an optimal a priori error estimate of unilateral contact problem frictionless between deformable body and rigid one.

For the numerical point of view, we observe an optimality of this method for both variables, the displacement and the Lagrange multipliers. In our experiments, we use a NURBS of degree 2 for the primal space and B-Spline of degree 0 for the dual space. Thanks to this choice of approximation spaces, we observe a stability of the Lagrange multipliers and a well approximation of the pressure in two-dimensional case and we observe a sub-optimality as a pre-asymptotic convergence in three-dimensional case. The sub-optimality observed in three-dimensional case may be due to the coarse mesh used. This NURBS based contact formulation seems to provide a robust description of large deformation.

\section{Acknowledgements}

This work has been partially supported by Michelin under the contract A10-4087. A. Buffa acknowledges the support of the ERC Advanced grant no. 694515 and of the PRIN-MIUR project "Metodologie innovative nella modellistica differenziale numerica". 


\section{Appendix 1.}

In this appendix, we provide the ingredients needed to fully discretise the problem (19) as well as its large deformation version that we have used in Section 4. First we introduce the contact status, an active-set strategy for the discrete problem, and then the fully discrete problem. For the purpose of this appendix, we take notations suitable to large deformation and denote by $g_{n}$ the distance between the rigid and the deformable body. In small deformation, it holds $g_{n}(u)=u \cdot n$.

\section{Contact status}

Let us first deal with the contact status. The active-set strategy is defined in $[16,15]$ and is updated at each iteration of Newton. Due to the deformation, parts of the workpiece may come into contact or conversely may loose contact. This change of contact status changes the loading that is applied on the boundary of the mesh. This method is used to track the location of contact during the change in boundary conditions.

Let $K$ be a control point of the B-Spline space $(7)$, let $\left(\Pi_{\lambda}^{h} \cdot\right)_{K}$ be the local projection defined in (8) and let $P\left\{\lambda_{K},\left(\Pi_{\lambda}^{h} g_{n}\right)_{K}\right\}$ be he operator defined component wise by:

- $\lambda_{K}=0$,

(1) if $\left(\Pi_{\lambda}^{h} g_{n}\right)_{K} \geq 0$, then $P\left\{\lambda_{K},\left(\Pi_{\lambda}^{h} g_{n}\right)_{K}\right\}=0$,

(2) if $\left(\Pi_{\lambda}^{h} g_{n}\right)_{K}<0$, then $P\left\{\lambda_{K},\left(\Pi_{\lambda}^{h} g_{n}\right)_{K}\right\}=\left(\Pi_{\lambda}^{h} g_{n}\right)_{K}$,

- $\lambda_{K}<0$,

(3) $P\left\{\lambda_{K},\left(\Pi_{\lambda}^{h} g_{n}\right)_{K}\right\}=\left(\Pi_{\lambda}^{h} g_{n}\right)_{K}$.

The optimality conditions are then written as $P\left\{\lambda_{K},\left(\Pi_{\lambda}^{h} g_{n}\right)_{K}\right\}=0$. So in the case (1), the constraints are inactive and in the case (2) and (3), the constraints are active.

\section{Discrete problem}

The space $V^{h}$ is spanned by mapped NURBS of type $\hat{N}_{i}^{p}(\boldsymbol{\zeta}) \circ \varphi_{0, \Gamma_{C}}^{-1}$ for $\boldsymbol{i}$ belonging to a suitable set of indices. In order to simplify and reduce our notation, we call $A$ as the running index, of control points associated with the surface $\Gamma_{C}, A=0 \ldots \mathcal{A}$ on this basis and set:

$$
V^{h}=\operatorname{Span}\left\{N_{A}(x), \quad A=0 \ldots \mathcal{A}\right\} \cap V .
$$

Now, we express quantities on the contact interface $\Gamma_{C}$ as follows:

$$
\left.u\right|_{\Gamma_{C}}=\sum_{A=1}^{\mathcal{A}} u_{A} N_{A},\left.\quad \delta u\right|_{\Gamma_{C}}=\sum_{A=1}^{\mathcal{A}} \delta u_{A} N_{A} \quad \text { and } \quad x=\sum_{A=1}^{\mathcal{A}} x_{A} N_{A},
$$

where $C_{A}, u_{A}, \delta u_{A}$ and $x_{A}=\varphi\left(X_{A}\right)$ are the related reference coordinate, displacement, displacement variation and current coordinate vectors.

By substituting the interpolations, the normal gap becomes:

$$
g_{n}=\left[\sum_{A=1}^{\mathcal{A}} C_{A} N_{A}(\zeta)+\sum_{A=1}^{\mathcal{A}} u_{A} N_{A}(\zeta)\right] \cdot n .
$$


In the previous equation, $\zeta$ are the parametric coordinates of the generic point on $\Gamma_{C}$. To simplify, we denote for the next of the purpose $\mathcal{D} g_{n}[\delta u]=\delta g_{n}$. The virtual variation follows as

$$
\delta g_{n}=\left[\sum_{A=1}^{\mathcal{A}} \delta u_{A} N_{A}(\zeta)\right] \cdot n .
$$

In order to formulate the problem in matrix form, the following vectors are introduced:

$$
\delta \boldsymbol{u}=\left[\begin{array}{c}
\delta u_{1} \\
\vdots \\
\delta u_{\mathcal{A}}
\end{array}\right], \quad \boldsymbol{\Delta}=\left[\begin{array}{c}
\Delta u_{1} \\
\vdots \\
\Delta u_{\mathcal{A}}
\end{array}\right], \quad \boldsymbol{N}=\left[\begin{array}{c}
N_{1}(\zeta) n \\
\vdots \\
N_{\mathcal{A}}(\zeta) n
\end{array}\right] .
$$

With the above notations, the virtual variation and the linearized increments can be written in matrix form as follow:

$$
\delta g_{n}=\delta \boldsymbol{u}^{T} \boldsymbol{N}, \quad \Delta g_{n}=\boldsymbol{N}^{T} \Delta \boldsymbol{u} .
$$

The contact contribution of the virtual work is expressed as follows:

$$
\delta W_{c}=\int_{\Gamma_{C}} \lambda \delta g_{n} \mathrm{~d} \Gamma+\int_{\Gamma_{C}} \delta \lambda g_{n} \mathrm{~d} \Gamma .
$$

The discretized contact contribution can be expressed as follows:

$$
\begin{aligned}
\delta W_{c} & =\int_{\Gamma_{C}} \sum_{K=1}^{\mathcal{K}} \lambda_{K} B_{K} \delta g_{n} \mathrm{~d} \Gamma+\int_{\Gamma_{C}} \sum_{K=1}^{\mathcal{K}} \delta \lambda_{K} B_{K} g_{n} \mathrm{~d} \Gamma, \\
& =\sum_{K} \lambda_{K} \int_{\Gamma_{C}} B_{K} \delta g_{n} \mathrm{~d} \Gamma+\delta \lambda_{K} \int_{\Gamma_{C}} B_{K} g_{n} \mathrm{~d} \Gamma, \\
& =\sum_{K} \lambda_{K} \int_{\Gamma_{C}} B_{K} \delta g_{n} \mathrm{~d} \Gamma+\delta \lambda_{K} \int_{\Gamma_{C}} B_{K} g_{n} \mathrm{~d} \Gamma, \\
& =\sum_{K}\left(\lambda_{K}\left(\Pi_{\lambda}^{h} \delta g_{n}\right)_{K}+\delta \lambda_{K}\left(\Pi_{\lambda}^{h} g_{n}\right)_{K}\right) K_{K},
\end{aligned}
$$

where $K_{K}=\int_{\Gamma_{C}} B_{K} \mathrm{~d} \Gamma$.

Indeed, we need to resolve a variational inequality. Using the contact status, we distinguish between the constraints on the control point $K$ are actives, i.e. when the contact occurs, and the constraints on the control point $K$ are inactives, i.e. when we loose the contact.

Using active-set strategy on the local gap $\left(\Pi_{\lambda}^{h} g_{n}\right)_{K}$ and $\lambda_{K}$, it holds:

$$
\delta W_{c}=\sum_{K, a c t}\left(\lambda_{K}\left(\Pi_{\lambda}^{h} \delta g_{n}\right)_{K}+\delta \lambda_{K}\left(\Pi_{\lambda}^{h} \delta g_{n}\right)_{K}\right) K_{K}
$$

At the discrete level we proceed as follows:

- We have $\sum_{K, \text { inact }} \delta \lambda_{K}\left(\Pi_{\lambda}^{h} g_{n}\right)_{K} \leq 0, \forall \delta \lambda_{K}$, i.e. $\left(\Pi_{\lambda}^{h} g_{n}\right)_{K} \geq 0$ a.e. on inactive part.

- On the active part, it holds $\sum_{K, \text { act }} \delta \lambda_{K}\left(\Pi_{\lambda}^{h} g_{n}\right)_{K}=0, \forall \delta \lambda_{K}$, i.e. $\left(\Pi_{\lambda}^{h} g_{n}\right)_{K}=0$ a.e.. 
- We impose too, $\sum_{K, \text { inact }} \lambda_{K}\left(\Pi_{\lambda}^{h} \delta g_{n}\right)_{K}=0, \forall\left(\Pi_{\lambda}^{h} \delta g_{n}\right)_{K}$, i.e. $\lambda_{K}=0$ a.e. on inactive boundary.

For the further developments it is convenient to define the vector of the virtual variations and linearizations for the Lagrange multipliers:

$$
\delta \boldsymbol{\lambda}=\left[\begin{array}{c}
\delta \lambda_{1} \\
\vdots \\
\delta \lambda_{\mathcal{K}}
\end{array}\right], \quad \Delta \boldsymbol{\lambda}=\left[\begin{array}{c}
\Delta \lambda_{1} \\
\vdots \\
\Delta \lambda_{\mathcal{K}}
\end{array}\right], \quad \boldsymbol{N}_{\lambda, g}=\left[\begin{array}{c}
\left(\Pi_{\lambda}^{h} g_{n}\right)_{1, a c t} K_{1, a c t} \\
\vdots \\
\left(\Pi_{\lambda}^{h} g_{n}\right)_{\mathcal{K}, a c t} K_{\mathcal{A}, a c t}
\end{array}\right], \quad \boldsymbol{B}_{\lambda}=\left[\begin{array}{c}
B_{1}(\zeta) \\
\vdots \\
B_{\mathcal{K}}(\zeta)
\end{array}\right] .
$$

In the matrix form, it holds:

$$
\delta W_{c}=\delta \boldsymbol{u}^{T} \int_{\Gamma_{C}}\left(\sum_{K, a c t} B_{K} \lambda_{K}\right) \boldsymbol{N} \mathrm{d} \Gamma+\delta \boldsymbol{\lambda}^{T} \boldsymbol{N}_{\lambda, g},
$$

and the residual for Newton-Raphson iterative scheme is obtained as:

$$
R=\left[\begin{array}{l}
R_{u} \\
R_{\lambda}
\end{array}\right]=\left[\begin{array}{c}
\int_{\Gamma_{C}}\left(\sum_{K, a c t} B_{K} \lambda_{K}\right) \boldsymbol{N} \mathrm{d} \Gamma \\
\boldsymbol{N}_{\lambda, g}
\end{array}\right]
$$

The linearization yields:

$$
\Delta \delta W_{c}=\int_{\Gamma_{C}} \Delta \lambda \delta g_{n} \mathrm{~d} \Gamma+\int_{\Gamma_{C}} \delta \lambda \Delta g_{n} \mathrm{~d} \Gamma
$$

The active-set strategy and the discretised of contact contribution can be expressed as follows:

$$
\begin{aligned}
\Delta \delta W_{c} & =\sum_{K, a c t} \sum_{A} \int_{\Gamma_{C}} \Delta \lambda_{K} B_{K} N_{A} \delta u_{A} \cdot n \mathrm{~d} \Gamma+\int_{\Gamma_{C}} \delta \lambda_{K} B_{K} N_{A} \Delta u_{A} \cdot n \mathrm{~d} \Gamma \\
& =\delta \boldsymbol{u}^{T} \int_{\Gamma_{C}, a c t} \boldsymbol{N} \boldsymbol{B}_{\lambda}^{T} \mathrm{~d} \Gamma \Delta \boldsymbol{\lambda}+\delta \boldsymbol{\lambda}^{T} \int_{\Gamma_{C}, a c t} \boldsymbol{B}_{\lambda} \boldsymbol{N}^{T} \mathrm{~d} \Gamma \Delta \boldsymbol{u} .
\end{aligned}
$$




\section{References}

[1] P. Alart and A. Curnier, A generalized Newton method for contact problems with friction, Journal de Mécanique Théorique et Appliquée, 7 (1988), pp. 67-82.

[2] Y. Bazilevs, L. Beirão da Veiga, J. A. Cottrell, T. J. R. Hughes, and G. SanGALLI, Isogeometric analysis: Approximation, stability and error estimates for h-refined meshes, Mathematical Models and Methods in Applied Sciences, 16 (2006), pp. 1031-1090.

[3] L. Beirão da Veiga, A. Buffa, G. Sangalli, And R. VÁzquez, Mathematical analysis of variational isogeometric methods, Acta Numerica, 23 (2014), pp. 157-287.

[4] F. Ben Belgacem and Y. Renard, Hybrid finite element methods for the Signorini problem, Mathematics of Computation, 72 (2003), pp. 1117-1145.

[5] D. Boffi, F. Brezzi, And M. Fortin, Mixed finite element methods and applications, Computational Mathematics, Springer, 2013.

[6] E. Brivadis, A. Buffa, B. Wohlmuth, And L. Wunderlich, Isogeometric mortar methods, Computer Methods in Applied Mechanics and Engineering, 284 (2015), pp. 292 319 .

[7] P. Coorevits, P. Hild, K. Lhalouani And T. Sassi, , Mixed finite element methods for unilateral problems: convergence analysis and numerical studies, Mathematics of Computation, 71 (2002), pp. 67-82

[8] L. De Lorenzis, J. Evans, T. Hughes, And A. Reali, Isogeometric collocation: Neumann boundary conditions and contact, Computer Methods in Applied Mechanics and Engineering, 284 (2015), pp. 21-54.

[9] L. De Lorenzis, P. Wriggers, and T. J. Hughes, Isogeometric contact: A review, GAMM-Mitteilungen, 37 (2014), pp. 85-123.

[10] L. De Lorenzis, P. Wriggers, and G. Zavarise, A mortar formulation for 3D large deformation contact using NURBS-based isogeometric analysis and the augmented Lagrangian method, Springer-Verlag, 49 (2012), pp. 1-20.

[11] G. Drouet And P. Hild, Optimal convergence for discrete variational inequalities modelling Signorini contact in $2 D$ and $3 D$ without additional assumptions on the unknown contact set, SIAM Journal on Numerical Analysis, 53 (2015), pp. 1488-1507.

[12] J. Haslinger, I. HlaváČek, and J. NeČas, Handbook of Numerical Analysis (eds. P.G. Ciarlet and J.L. Lions), vol. IV, North Holland, 1996, ch. 2. "Numerical methods for unilateral problems in solid mechanics", pp. 313-385.

[13] H. HeRTz, Üeber die berührung fester elastischer körper, Journal für die reine und angewandte Mathematik, 92 (1882), pp. 156-171.

[14] P. Hild And P. Laborde, Quadratic finite element methods for unilateral contact problems, Applied Numerical Mathematics, 41 (2002), pp. 401-421. 
[15] S. Hüeber, G. Stadler, And B. I. Wohlmuth, A primal-dual active set algorithm for three-dimensional contact problems with coulomb friction, SIAM Journal on Scientific Computing, 30 (2008), pp. 572-596.

[16] S. Hüeber And B. I. Wohlmuth, A primal-dual active set strategy for non-linear multibody contact problems, Computer Methods in Applied Mechanics and Engineering, 194 (2005).

[17] T. J. R. Hughes, J. A. Cottrell, And Y. Bazilev, Isogeometric analysis: Cad, finite elements, NURBS, exact geometry and mesh refinement, Computer Methods in Applied Mechanics and Engineering, 194 (2005), pp. 4135-4195.

[18] A. Konyukhov and K. Schweizerhof, Computational contact mechanics, vol. 67, Applied and Computational Mechanics, 2013.

[19] T. A. Laursen, Computational contact and impact mechanics, Springer-Verlag, Berlin, 2003.

[20] J. L. LiOns AND E. MAGEnEs, Non-homogeneous boundary value problems and applications, Springer-Verlag, Berlin, New York, 1972.

[21] M. S. Pauletti, M. Martinelli, N. Cavallini, and P. Antolin, Igatools: An isogeometric analysis library, SIAM Journal on Scientific Computing, 37 (2015).

[22] A. Popp, B. I. Wohlmuth, M. W. Gee, And W. A. Wall, Dual quadratic mortar finite element methods for $3 D$ finite deformation contact, SIAM Journal on Scientific Computing, 34 (2012), pp. B421-B446.

[23] K. Poulios And Y. Renard, An unconstrained integral approximation of large sliding frictional contact between deformable solids, Computers and Structures, 153 (2015), pp. 7590.

[24] A. Seitz, P. Farah, J. Kremheller, B. I. Wohlmuth, W. A. Wall, and A. Popp, Isogeometric dual mortar methods for computational contact mechanics, Computer Methods in Applied Mechanics and Engineering, 301 (2016), pp. 259-280.

[25] I. Temizer, P. Wriggers, And T. Hughes, Contact treatment in isogeometric analysis with NURBS, Computer Methods in Applied Mechanics and Engineering, 200 (2011), pp. $1100-1112$.

[26] I. Temizer, P. Wriggers, And T. Hughes, Three-dimensional mortar-based frictional contact treatment in isogeometric analysis with NURBS, Computer Methods in Applied Mechanics and Engineering, 209-212 (2012), pp. 115-128.

[27] B. I. Wohlmuth, A mortar finite element method using dual spaces for the Lagrange multiplier, SIAM Journal on Numerical Analysis, 38 (2000), pp. 989-1012.

[28] P. Wriggers, Computational contact mechanics (Second Edition), Wiley, 2006.

[29] G. ZAVARISE AND L. D. LoREnZIS, The node-to-segment algorithm for $2 D$ frictionless contact: Classical formulation and special cases, Computer Methods in Applied Mechanics and Engineering, (2009). 